\title{
A DFT+U study of the oxidation of cobalt nanoparticles: Implications for biomedical applications
}

\author{
Barbara Farkaš, David Santos-Carballal, Abdelaziz Cadi-Essadek, Nora H. de Leeuw* \\ School of Chemistry, Cardiff University, Main Building, Park Place, Cardiff CF10 3AT, UK
}

\section{A R T I C L E I N F O}

\section{Keywords:}

Computer modelling

DFT

Cobalt nanoparticles

Surface oxidation

\begin{abstract}
A B S T R A C T
Nanomaterials - magnetic nanoparticles in particular have been shown to have significant potential in cancer theranostics, where iron oxides are commonly the materials of choice. While biocompatibility presents an advantage, the low magnetisation is a barrier to their widespread use. As a result, highly magnetic cobalt nanoparticles are attracting increasing attention as a promising alternative. Precise control of the physiochemical properties of such magnetic systems used in biomedicine is crucial, however, it is difficult to test their behaviour in vivo. In the present work, density functional theory calculations with the Dudarev approach (DFT+U) have been used to model the adsorption of oxygen on low Miller index surfaces of the hexagonal phase of cobalt. In vivo conditions of temperature and oxygen partial pressure in the blood have been considered, and the effects of oxidation on the overall properties of cobalt nanoparticles are described. It is shown that oxygen adsorbs spontaneously on all surfaces with the formation of non-magnetic cobalt tetroxide, $\mathrm{Co}_{3} \mathrm{O}_{4}$, at body temperature, confirming that, despite their promising magnetic properties, bare cobalt nanoparticles would not be suitable for biomedical applications. Surface modifications could be designed to preserve their favourable characteristics for future utilisation.
\end{abstract}

\section{Introduction}

Cancer remains one of the most devastating contemporary diseases with worldwide cases predicted to increase by $50 \%$ in the next two decades [1]. In spite of improved detection techniques and therapies, limited efficiency and damage of adjacent tissue remain a huge concern [2]. In order to destroy only targeted cancer cells whilst leaving the surroundings intact, more intelligent approaches need to be developed beyond drug injections and chemotherapy. Nanomaterials are offering a promising solution, due to their exceptional properties, changeable size, and simple modifications, providing controllable means for targeting and interacting with specific cells [3].

Metal nanoparticles are especially interesting - with dimensions proportional to the size of entities controlling body processes, and through the easy manipulation of their optical, mechanical, magnetic, and electronic properties, they can be readily utilised in biomedicine [4], transforming outside-in treatments to inside-out. Perhaps the best example of their efficacy is hyperthermia therapy, where implementation of magnetic nanoparticles moves the source of heat into the targeted cells, minimising the temperature gradient in surrounding tissues [5]. However, finding the perfect match between nanoparticles' type, size, and desired properties is still the biggest challenge for this new area of research.
Magnetite $\left(\mathrm{Fe}_{3} \mathrm{O}_{4}\right)$ nanoparticles are the most thoroughly tested, since they are highly biocompatible. However, due to their low saturation magnetisation, relatively large nanoparticles and strong external fields, usually unfavourable for humans, are required to achieve sufficient effects [6]. Transition metal particles have much higher saturation magnetisation, which means that higher heating rates could be achieved for the same or even lower concentrations compared to the corresponding oxides. To date, none of the studied materials has met the requirements of hyperthermic treatments at reasonable concentrations to target even the smallest tumours [7]. Unfortunately, intensive interaction of the metals with compounds present in vivo hinders their otherwise advantageous magnetic properties.

Recent studies have revealed promising shift when doping magnetite with transition metals, e.g. cobalt, resulting in smaller nanoparticles with simultaneous improvement of the magnetic properties: up to fourteen-fold increase in MRI contrast and a fourfold enhancement in hyperthermic effects $[8,9]$. However, despite these major advances, the overall efficacy of (doped) magnetite is still insufficient, and pure metallic nanoparticles, although reactive, may provide an alternative solution as their performance exceeds by far any metal oxide. Therefore, there is a growing interest in new modification processes which will provide protection of the metal nanoparticles against oxidation, while retaining their magnetic properties. Two potential candidates with the

\footnotetext{
* Corresponding author.

E-mail address: deleeuwn@cardiff.ac.uk (N.H. de Leeuw).
} 
highest heating power are iron and cobalt. Although iron has better heating effects [7], the maximum occurs for higher diameters compared to cobalt and, once coupled with modification agents, larger particles would quickly be endocytosed by macrophages and removed from the body. Cobalt therefore offers better functionalisation, which can be conducted to a greater extent and coupled with additional features like drug delivery.

To date, cobalt nanoparticles have mainly been investigated for their magnetic [10] and catalytic [11] applications, whereas relatively less effort has been expended on their implementation in biomedical applications, owing to the possible toxicity of elemental cobalt [12]. Despite intensive research, toxicity mechanisms for nanoparticles, and the exact effects of cobalt in human organism, are still not completely understood [13]. What is well known is the oxidation of cobalt at room temperature, with both cobalt (II) oxide and cobalt (II,III) tetroxide losing their antiferromagnetism above 291 and $40 \mathrm{~K}$, respectively, and becoming nonmagnetic [14]. Thus, if oxidation of the cobalt nanoparticles was to occur in the presence of oxygen in vivo, e.g. in blood, the formation of oxides would cause the loss of the highly desirable magnetic properties and the nanoparticles could therefore not be used in biomedical treatments without additional modifications.

As adsorption of oxygen on metal surfaces is highly relevant to general material processes, such as catalysis and corrosion, it has been extensively studied both experimentally [15-17] and theoretically [18-21]. Oxidation has been associated with oxygen adsorbed on the surface, the formation of surface oxide films, and the formation of bulk metal oxides of transition metals, nominally ranging from Sc $[22,23]$ to $\mathrm{Zn}[24,25]$, and rare earth metals such as La $[26,27]$ and $\mathrm{U}[28,29]$. Even on the noblest of metals, Pd, Ag, Pt, and Au, oxidised surfaces or islands have been implicated in the observed oxidation activity [30-35]. Cobalt is not an exception and, as it has been recognised as a good catalyst in the Fischer-Tropsch synthesis, a number of works have been devoted to explore its oxidation behaviour in the cubic phase [36-38].

However, to the best of our knowledge, apart from the Co (0001) surface $[39,40]$, no theoretical work on the interaction of oxygen with the hexagonal phase of cobalt has been published. Therefore, in the present work the oxygen adsorption on hexagonal closed packed (hcp) cobalt considering five low Miller index surfaces has been systematically analysed to confirm aforementioned hypothesis. Coverage-dependent adsorption properties ranging from energetics and site preference to atomic and electronic structures (e.g. bond distances, densities of state, charge density differences) are discussed in comparison with available experimental data. Thermodynamic phase diagrams based on ab initio thermodynamics have been proposed, taking into account the effects of temperature and pressure. Our work aims to provide insight into two issues:

1) Is the oxidation spontaneous and should it be prioritised over the possible toxicity of cobalt nanoparticles?

2) What should be done to overcome either the problem of loss of magnetisation through the spontaneous adsorption of oxygen or any toxic effects of cobalt in vivo (if oxidation does not happen) to be able to utilise cobalt's promising magnetic properties in biomedicine?

\section{Computational methods}

The Vienna Ab-initio Simulation Package (VASP) code [41] has been used to carry out spin-polarised calculations within the usual KohnSham (KS) implementation of the density functional theory (DFT) [42]. The generalised gradient approximation (GGA) was employed to approximate the exchange-correlation functional using parametrisation developed by Perdew, Burke, and Ernzerhof (PBE) [43]. The long-range dispersion interactions were added through the DFT-D3 method with Becke-Johnson damping [44] as their inclusion is necessary for the correct description of the surface properties [45]. The core electrons up to and including the $3 p$ levels of $\mathrm{Co}$ and the $1 \mathrm{~s}$ levels of $\mathrm{O}$ were kept frozen and their interaction with the valence electrons was described by the projector augmented wave (PAW) method [46].

As our primary interest is the partial oxidation of cobalt metal surfaces and the resulting changes in the energetic and magnetic characteristics of the material, the DFT+U method [47] using the Dudarev approach was employed, as implemented in the VASP software, rather than standard GGA which is known to fail in the description of the energies and electronic properties of the localised orbitals of transition metals and their oxides $[48,49]$. The $U$ here is the effective Hubbard $U_{\text {eff }}=U-J$, where $J$ is equal to zero, and a value of $3.0 \mathrm{eV}$ was adopted for the $3 \mathrm{~d}$ orbitals of $\mathrm{Co}$. This $U$ value was obtained through a systematic study of oxidation energies for hep cobalt to $\mathrm{CoO}$ and $\mathrm{Co}_{3} \mathrm{O}_{4}$, and $\mathrm{CoO}$ to $\mathrm{Co}_{3} \mathrm{O}_{4}$ as the Hubbard Hamiltonian is known to affect differently the relative energies of transitions metals and their oxides [50,51]. At this point it is worth noting that although $U=3.0 \mathrm{eV}$ was developed based on oxidation energy arguments, it is also consistent with the value required to reproduce accurately the electronic structure of cobalt and its oxides [52]. Thus, we are confident of our predicted energies and electronic properties for the partial oxidation of the major surfaces of hexagonal Co. Details are provided in the electronic Supplementary Information, SI.

\subsection{Bulk}

Bulk calculations were conducted on an hcp cell (P63/mmc space group) containing two cobalt atoms, both of which were fully relaxed until the required accuracy was reached. The hexagonal phase was considered rather than the cubic one since the hcp $\rightarrow$ fcc phase transition occurs at high temperatures $\left(>450^{\circ} \mathrm{C}\right)$ which are not relevant in the biomedical field [53]. Calculations were carried out in reciprocal space with a $17 \times 17 \times 9$ k-point mesh and a cut-off energy of $400 \mathrm{eV}$ to determine the number of plane-waves required to describe the system. The conjugate gradient technique, with a force convergence criterion of $0.01 \mathrm{eV} / \AA$, has been used to perform geometry optimisations.

\subsection{Surfaces}

The METADISE code [54] was employed to construct the structures of the five inequivalent low Miller index surfaces: (0001), (0110), (1011), (1120), and (112̄1). The studied surfaces were modelled as a slab of material with periodic boundary conditions and a vacuum in the direction orthogonal to the surface. The optimised slab model consisted of four layers with the bottom two layers fixed at their bulk equilibrium positions and representing the bulk material, whereas the top two layers were allowed to fully relax. $16 \AA$ of vacuum thickness was found to be sufficient to prevent interaction between two vertical images for all surfaces. Supercells were constructed of $3 \times 3$ for (0001), $3 \times 2$ for $(01 \overline{1} 0)$, $2 \times 2$ for $(11 \overline{2} 0)$ and $(11 \overline{2} 1)$ surfaces with $5 \times 5 \times 1$ k-point mesh, and $3 \times 2$ supercell with $5 \times 4 \times 1$ mesh for (1011) surface due to the symmetry breaking.

To characterise the surfaces, surface energies, $\gamma$, as a measure of the thermodynamic stability have been calculated through the following equations:

$\gamma_{\mathrm{u}}=\frac{E_{\text {unrelaxedslab }}^{\mathrm{DFT}}-\mathrm{n} \times E_{\text {bulk }}^{\mathrm{DFT}}}{2 A_{\text {slab }}}$,

$\gamma_{\mathrm{r}}=\frac{E_{\text {relaxedslab }}^{\mathrm{DFT}}-\mathrm{n} \times E_{\text {bulk }}^{\mathrm{DFT}}}{A_{\text {slab }}}-\gamma_{\mathrm{u}}$

where $\gamma_{\mathrm{u}}$ and $\gamma_{\mathrm{r}}$ are the surface energies before and after relaxation, $E_{\text {unrelaxedslab }}^{\text {DFT }}, E_{\text {relaxedslab }}^{\text {DFT }}$, and $E_{\text {bulk }}^{\text {DFT }}$ the DFT energies of the unrelaxed and relaxed slab, and bulk, respectively, $A_{\text {slab }}$ the surface area, and $n$ the ratio between the number of Co atoms in the slab and the number of Co atoms in the bulk. The bulk model considered here is the same twoatom hcp cell used for calculations of fundamental hcp Co properties. The lowest surface energy after the relaxation represents the most stable 
Table 1

Calculated cell parameters, magnetic moment, and bulk modulus for the hcp Co bulk phase and comparison with previous studies.

\begin{tabular}{|c|c|c|c|c|c|c|c|}
\hline & & \multirow[t]{2}{*}{ This work } & \multirow[t]{2}{*}{ Experiment [59] } & \multicolumn{3}{|l|}{ Theory } & \multirow[t]{2}{*}{ Percent error } \\
\hline & & & & GGA [64] & GGA+U [65] & HSE06 [64] & \\
\hline \multirow[t]{3}{*}{ Cell parameter } & $a, b / \AA$ & 2.444 & 2.51 & 2.496 & - & 2.508 & 2.6 \\
\hline & $c / \AA$ & 4.051 & 4.07 & 4.030 & - & 4.470 & 0.5 \\
\hline & $\mathrm{c} / \mathrm{a}$ & 1.657 & 1.62 & 1.615 & 1.60 & 1.782 & -2.3 \\
\hline \multicolumn{2}{|c|}{ Magnetic moment $/ \mu_{B}$} & 1.760 & 1.72 & 1.59 & 1.725 & 2.09 & -2.3 \\
\hline \multicolumn{2}{|c|}{ Bulk modulus/GPa } & 182.532 & 191 & 207 & 166.3 & 107 & 4.4 \\
\hline
\end{tabular}

surface. Since it has been shown that the surface energy is insensitive to variations in temperature as the vibrations of the slab differ slightly from the vibrations of the bulk [19], that contribution was not considered. Surface energies have then been implemented in the Wulffmaker software [55] to obtain the Wulff morphology [56] of nanoparticles.

The second parameter calculated to characterise each surface was the electronic work function, $\phi$, which is the energy required to completely withdraw an electron from the solid. It was obtained as the difference between the vacuum electrostatic potential energy, $E_{\mathrm{vac}}$, and the energy of the Fermi level, $E_{\mathrm{F}}$.

\subsection{Adsorption}

Adsorption of oxygen on-surface and sub-surface was carried out on all five surfaces. The initial position of the oxygen atom on-surface was $2.2 \AA$ above the surface, equalling cobalt-oxygen distances in cobalt oxides [57]. For each site on-surface, the oxygen atom and the top two layers of the slab were relaxed, whereas for sub-surface adsorption, slab models of 6 layers were used with the top 4 allowed to relax. The adsorption energy, $E_{\text {ads }}$, was calculated as follows:

$E_{\text {ads }}=E_{\text {surface }+\mathrm{O}}^{\mathrm{DFT}}-\left(E_{\text {surface }}^{\mathrm{DFT}}+E_{\mathrm{O}}^{\mathrm{DFT}}\right)$

where $E_{\text {surface+O }}^{\mathrm{DFT}}, E_{\text {surface}}^{\mathrm{DFT}}$, and $E_{\mathrm{O}}^{\mathrm{DFT}}$ are the DFT energies of the system with adsorbed oxygen, the clean surface, and the oxygen atom, respectively. A negative adsorption energy represents spontaneous oxidation, with the lowest adsorption energy indicating the most favourable adsorption site. Work functions of the surfaces after the oxygen adsorption have been calculated as for the clean surfaces. Increased coverages have been performed afterwards for a certain number of possible configurations, with the adsorption energies calculated in the same way, and the cumulative adsorption energies, $E_{\text {ads,cum }}$, calculated as:

$E_{\text {ads,cum }}=E_{\text {surface+nO }}^{\mathrm{DFT}}-\left(E_{\text {surface }+(\mathrm{n}-1) \mathrm{O}}^{\mathrm{DFT}}+E_{\mathrm{O}}^{\mathrm{DFT}}\right)$

where $E_{\mathrm{surface}+\mathrm{nO}}^{\mathrm{DFT}}, E_{\mathrm{surface}+(\mathrm{n}-1) \mathrm{O}}^{\mathrm{DFT}}$, and $E_{\mathrm{O}}^{\mathrm{DFT}}$ are the DFT energies of the system with $n$ adsorbed oxygen atoms, the system with n-1 adsorbed oxygen atoms, and the oxygen atom, respectively.

As oxygen adsorbs or desorbs on the metal surface from or to the gas phase as $\mathrm{O}_{2}$, it is crucial to reference all DFT total energies to the groundstate energy of the $\mathrm{O}_{2}$ molecule in the gas phase. To achieve this, the $\mathrm{O}_{2}$ molecule has been modelled in vacuum. However, it should be noted that GGA leads to a large error in the binding energy of most covalent molecules, including the oxygen molecule, referred to in the literature as the over-binding $[20,58]$. To correct for this error, the DFT formation energy of the $\mathrm{O}_{2}$ molecule was compared to the empirically measured formation energy using thermochemistry tables [50]. In order to obtain accurate results, the zero-point energy was also considered. Finally, over-binding in the surface-adsorbed molecules could then be approximated by subtracting the empirically measured energy from the GGA calculated energy: $E_{\mathrm{OB}}=\Delta H_{f}^{\mathrm{DFT}}+E_{\mathrm{ZP}}-\Delta H_{f}^{\mathrm{exp}}$ where $E_{\mathrm{OB}}$ is the overbinding energy, $\Delta H_{f}^{\mathrm{DFT}}$ is the calculated formation enthalpy, $E_{\mathrm{ZP}}$ is the calculated zero-point energy, and $\Delta H_{f}^{\exp }$ is the experimentally measured formation enthalpy. DFT values obtained were $-5.92 \mathrm{eV}$ and $0.11 \mathrm{eV}$ for $\Delta H_{f}^{\mathrm{DFT}}$ and $E_{\mathrm{ZP}}$, respectively. Combined with $\Delta H_{f}^{\mathrm{exp}}$ of $-5.16 \mathrm{eV}$ [59], an over-binding energy of $-0.86 \mathrm{eV}$ was thereby detected, giving a correction of $-0.43 \mathrm{eV}$ per oxygen atom. The binding energy per oxygen atom obtained is $-2.58 \mathrm{eV}(-3.01 \mathrm{eV}$ without correction, corresponding well to other DFT works: $3.12 \mathrm{eV}$ [43], $3.04 \mathrm{eV}$ [18]) with an O-O bond length of $1.23 \AA$ and a vibrational frequency of $1567 \mathrm{~cm}^{-1}$. Agreement with experimental results $\left(-2.56 \mathrm{eV}, 1.21 \AA, 1580 \mathrm{~cm}^{-1}\right)$ [60] is now excellent. $E_{\mathrm{O}}^{\mathrm{DFT}}$ has been corrected in all following adsorption and thermodynamic processes where oxygen atoms were present.

Thermodynamics of different $O$ coverages in equilibrium with an oxygen reservoir was introduced by comparing the surface free energy, $\sigma$, at constant temperature, $T$, with changes in the conditions conducted in the oxygen chemical potential, $\mu_{\mathrm{O}}: \sigma(T, p)=\gamma_{\mathrm{r}}+\Delta \sigma(T, p)[61,62]$. To test the influence of the vibrational energy, the surface free energies of systems going from clean surfaces to surfaces with a full monolayer of oxygen are reported as a function of the oxygen chemical potential with and without inclusion of the vibrational contributions. Details are given in the SI.

\section{Results}

\subsection{Bulk}

The structure of the optimised hcp Co bulk is shown in Fig. S2, SI. Final lattice parameters are $a=2.444, b=2.444$, and $c=4.051 \AA$, resulting in a $c / a$ ratio of 1.657 . The calculated ratio deviates by less than $3 \%$ from experiment (1.62 [59]). A magnetic moment of $1.760 \mu_{\mathrm{B}}$ has been determined, corresponding well to the experimental value (1.72 $\mu_{B}$ [59]). Murnaghan's equation of state [63] has been used to calculate the bulk modulus and a value of $182.532 \mathrm{GPa}$ was obtained. Agreement with existing data is satisfactory, as shown in Table 1.

\subsection{Surfaces}

The surface slab models are represented and described in Fig. S3, SI, while Table 2 contains the calculated relaxed surface energies, $\gamma_{\mathrm{r}}$, interlayer relaxation displacements, $\Delta d_{12}, \Delta d_{23}$, and work functions, $\phi$, of five unequal low Miller index surfaces.

With surface energy of $2.11 \mathrm{Jm}^{-2}$, the (1011) surface is the most stable and therefore the most prominent, while the (0001) and (0110) surfaces have the highest energies. The final sequence in surface stability, according to the surface energies listed in Table 2 , is: $(10 \overline{1} 1)>(11 \overline{2} 1)$ $>(11 \overline{2} 0)>(01 \overline{1} 0)=(0001)$. The work function tends to be greater for dense crystal facets than for those with more open lattices [66], which agrees with the (0001) surface having the highest value of $5.64 \mathrm{eV}$. Two most open surfaces, (112̄0) and (112̄1), have low work functions of 3.80 and $3.57 \mathrm{eV}$, respectively. Other results are available only for the (0001) surface, with an experimental value of $5.55 \mathrm{eV}$ [67] and a DFT value obtained using the local density approximation of $5.62 \mathrm{eV}$ [68]. The percent error of our calculated value compared to the experiment is just $1.62 \%$ which can mainly be ascribed to the temperature difference (experiment carried out at $180 \mathrm{~K}$ ).

Changes in the distances between layers after relaxation are also provided in Table 2, with negative and positive values representing contraction and expansion, respectively, with respect to the unrelaxed structure. For the (0001) surface, $2.56 \%$ contraction in the spacing between first and second layer is observed. Quantitative structure determination with 


\section{Table 2}

Calculated relaxed surface energies $\left(\gamma_{\mathrm{r}}\right)$, interlayer relaxation rates $\left(\Delta d_{12}, \Delta d_{23}\right)$, and work functions $(\phi)$ of five low Miller index hcp Co surfaces together with available experimental, semi-empirical, and theoretical relaxed surface energies. The last column shows the top views with possible adsorption sites - for (0001) surface, darker atoms are atoms of the first layer, while for the rest of the surfaces black, dark grey, and light grey atoms represent the first atomic layer in the higher row, the first atomic layer in the lower row, and the atoms of the other layers, respectively. For semi-empirical and theoretical work, methods are given in brackets.

\begin{tabular}{|c|c|c|c|c|c|c|}
\hline surface & this work & $\begin{array}{c}\gamma_{\mathrm{r}} / \mathrm{J} \mathrm{m}^{-2} \\
\text { experimental / semi- } \\
\text { empirical work }\end{array}$ & $\begin{array}{c}\text { other theoretical } \\
\text { work }\end{array}$ & $\begin{array}{l}\Delta \mathrm{d}_{12} / \% \\
\Delta \mathrm{d}_{23} / \% \\
\end{array}$ & $\phi / \mathrm{eV}$ & adsorption positions* \\
\hline (0001) & 2.43 & $\begin{array}{c}2.55 \text { [69] (exp) } \\
2.99 \text { [72] (MEAM) } \\
1.16 \text { [73] (AMEAM) } \\
2.86 \text { [74] (EET) }\end{array}$ & $\begin{array}{l}2.78[70](G G A) \\
2.11[71](G G A)\end{array}$ & $\begin{array}{l}-2.56 \\
4.19\end{array}$ & 5.64 & \\
\hline$(01 \overline{1} 0)$ & 2.43 & 2.96 [74] (EET) & & $\begin{array}{l}0.77 \\
5.20\end{array}$ & 5.27 & \\
\hline$(10 \overline{1} 1)$ & 2.11 & 4.52 [72] (MEAM) & & $\begin{array}{l}4.65 \\
2.39\end{array}$ & 5.01 & \\
\hline$(11 \overline{2} 0)$ & 2.28 & $\begin{array}{c}4.81 \text { [72] (MEAM) } \\
3.41 \text { [74] (EET) }\end{array}$ & & $\begin{array}{l}4.42 \\
7.86\end{array}$ & 3.80 & \\
\hline$(11 \overline{2} 1)$ & 2.22 & 4.94 [72] (MEAM) & & $\begin{array}{c}0.26 \\
16.32\end{array}$ & 3.57 & \\
\hline \multicolumn{7}{|c|}{ 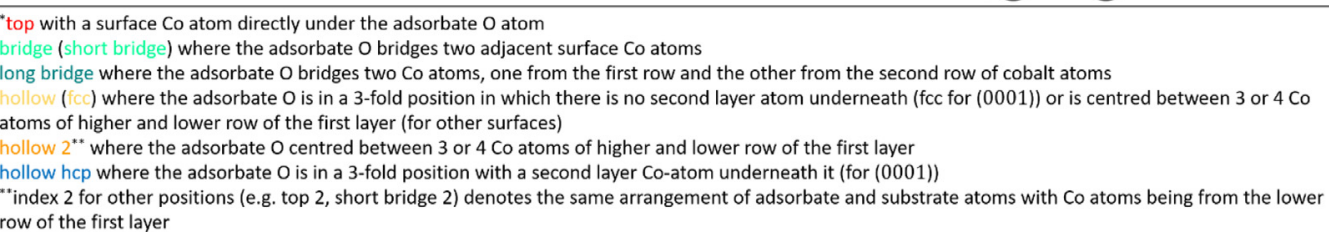 } \\
\hline
\end{tabular}

a low energy electron diffraction method [69] also predicts interlayerdistance contraction of $\sim 3 \%$ for the same pair of layers. The disparity in the values for the other distances can be ascribed to the choice of the slab model, but also to the diffraction method being exceptionally sensitive to the examination of high order layers [70]. Previous studies did not investigate other surfaces and a reference point is therefore missing.

Inconsistencies in surface energies and stability orders published previously make comparison with other works difficult. Nevertheless, the agreement with experimental results for the (0001) surface [71] is very good, thus providing confidence in the suitability of the parameters used. Discrepancies with available semi-empirical and computational results can be attributed to the choice of the slab model, exchangecorrelation functional, and, most importantly, inclusion of the longrange dispersion interactions in the present work which have been disregarded in earlier studies [72,73].

The Wulff morphology of nanoparticles constructed using the calculated relaxed surface energies is shown in Fig. 1. All five surfaces appear in the morphology.

\subsection{Adsorption}

3.3.1. Structure, adsorption energy, and properties at low coverages

An oxygen atom was initially placed at every possible adsorption site, defined in Table 2, on the five surfaces. Sub-surface sites are represented in Fig. S4, SI. To study the relative stabilities of different positions, adsorption energies have been calculated and are listed, along with structural parameters, in Table S1, SI. The adsorption energies of the most stable adsorption sites have been summarised in Table 3 for all five low Miller index surfaces.

Energetic and structural parameters: The calculated adsorption energies indicate that the threefold hollow fcc site is the most stable adsorption position for the (0001) surface with an adsorption energy of $-3.69 \mathrm{eV}$. The difference in the adsorption energy between two hollow positions (fcc-hcp $0.24 \mathrm{eV}$ ) is considerably less than between the hollow and top positions (fcc-top $1.46 \mathrm{eV}$ and hcp-top $1.21 \mathrm{eV}$ ) which is expected since hollow sites only differ from each other from the second layer down. When the oxygen is initially positioned in the bridge site, 
Table 3

Top and side view alongside calculated adsorption energies, $E_{\text {ads }}$, for the most stable adsorption sites on five low Miller index surfaces of hcp Co. Oxygen atoms are represented in red, with cobalt atoms of different layers being shown in shades of grey: the higher the layer, the darker the colour.

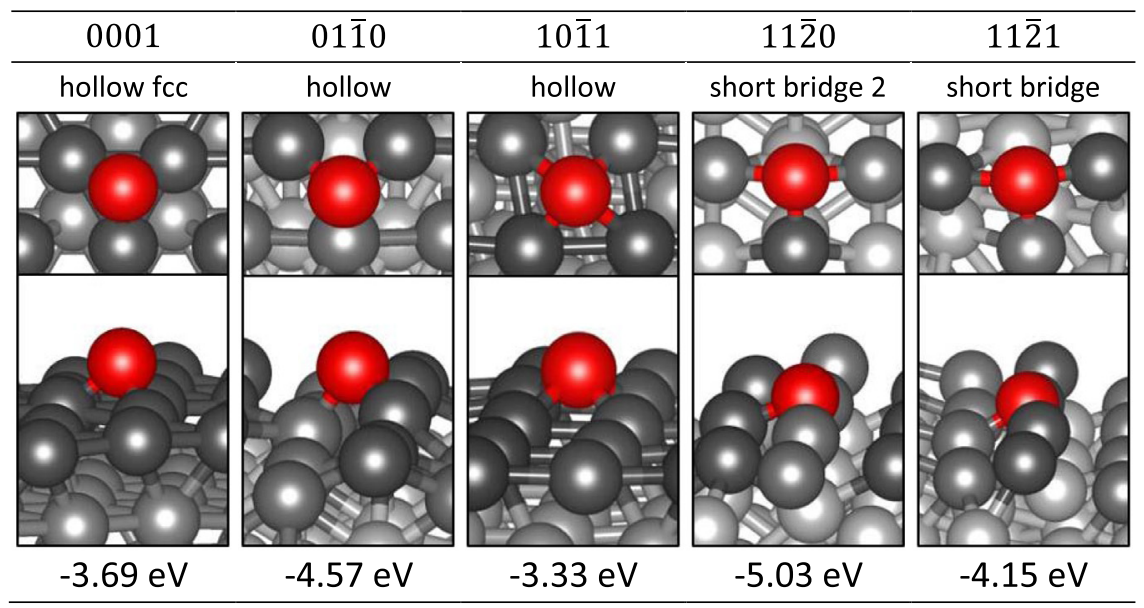
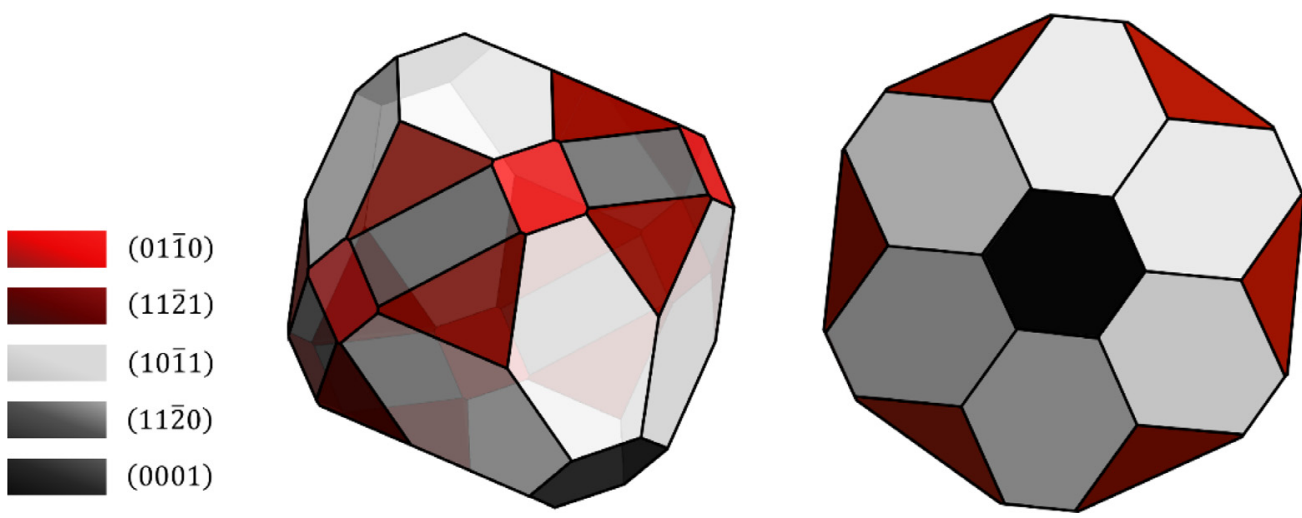

Fig. 1. Wulff morphology of hcp Co nanoparticles.

hollow fcc

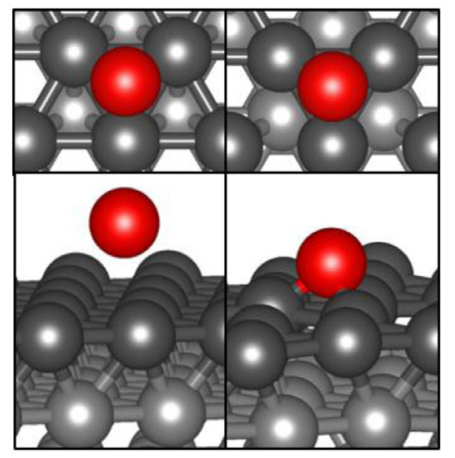

before

after

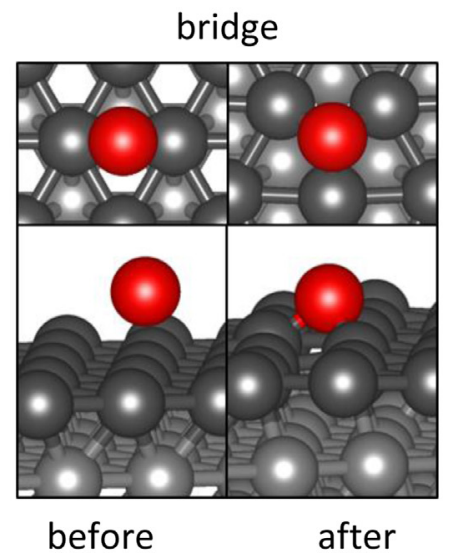

Fig. 2. Top and side views of the (0001) surface before and after the adsorption of one oxygen atom in hollow fcc and bridge positions. Oxygen atoms are represented in red, with cobalt atoms of different layers being shown in shades of grey: the higher the layer, the darker the colour. (For interpretation of the references to colour in this figure legend, the reader is referred to the web version of this article.)

it rearranges the original stacking of the (0001) surface during relaxation, Fig. 2, and ends up in the hollow fcc site; the calculated adsorption energy therefore cannot be compared to the rest of the positions. Three adsorption sites on the (0001) surface have been identified for sub-surface adsorption, i.e. two tetrahedral and one octahedral position, Fig. S4, SI. However, after geometry optimisation oxygen atom placed in the second tetrahedral position ended up on-surface. The most stable sub-surface position is tetrahedral, but it is $1.29 \mathrm{eV}$ less favourable than the most stable on-surface position. Thus, on-surface adsorption is preferred for single atom adsorption on the (0001) surface.

From a structural point of view, the oxygen-surface distances are in accord with the energetic parameters - the lower the adsorption energy, the closer the adsorbate is to the surface, Table S1, SI. When considering the Co-O distances, among the positions with the same coordination number of oxygen, the oxygen atom will be closest to the cobalt atoms in the site with the lowest energy. A complete list of the coordination 
Table 4

Calculated adsorption energies (with respect to atomic oxygen) and distances between the adsorbed oxygen atom and the closest Co atom in comparison with previous theoretical studies.

\begin{tabular}{|c|c|c|c|c|c|c|c|}
\hline & & & This work $^{\mathrm{a}}$ & Other theory & & & \\
\hline \multirow[t]{2}{*}{0001} & Hollow hcp & $\begin{array}{l}E_{\text {ads }} / \mathrm{eV} \\
d_{\mathrm{C}-\mathrm{O}} / \forall\end{array}$ & $\begin{array}{l}-5.35 \\
1.879\end{array}$ & $-5.52[74]$ & $\begin{array}{l}5.12[39] \\
1.86\end{array}$ & $-5.92[75]$ & $\begin{array}{l}5.34[76] \\
1.885\end{array}$ \\
\hline & Hollow fcc & $\begin{array}{l}E_{\mathrm{ads}} / \mathrm{eV} \\
d_{\mathrm{Co}-\mathrm{O}} / \forall\end{array}$ & $\begin{array}{l}-5.59 \\
1.872\end{array}$ & $-5.43[74]$ & & & \\
\hline
\end{tabular}

a Expressed with respect to atomic oxygen.

numbers for all sites is also given in Table S1, SI. The interlayer changes, induced by the oxygen adsorption, are as follows: inter-layer spacing between the first and second layer decreases (cobalt-oxygen attraction), and between the second and third layer increases (the third atomic layer is hardly affected by the oxygen atom).

Among six possible adsorption sites on the $(01 \overline{1} 0)$ surface, the only threefold site (hollow) is the most stable. Although there are three positions which have higher coordination numbers compared to the hollow site (top 2, L bridge, and hollow 2), the oxygen atom is unable to settle in the centre therefore forming bonds of different lengths with four or five closest cobalt atoms. The top 2 position, which closely resembles the hollow hcp of the (0001) surface, results in an adsorption energy that is for $1.26 \mathrm{eV}$ more favourable than the top site, as on the basis of the coordination number of oxygen. The oxygen coordination number has the same effect on the Co-O distances as in the case of the (0001) surface. There are two inequivalent sub-surface adsorption sites, Fig. S4, SI, and both are significantly less stable than even the least favourable on-surface adsorption site (top), thus significantly favouring any type of on-surface adsorption.

On the (1011) surface, the oxygen atom also prefers to adsorb in the hollow site. However, the adsorption energies for the two hollows are only $0.01-0.10 \mathrm{eV}$ lower than for any other position. These close values of the adsorption energies can be explained by the movement of the oxygen atom from all top and bridge starting positions towards the hollow sites during relaxation, which results in all positions having the same coordination number. Contrary to the (0001) surface, change in position is not followed by the shift of the first and second layer of cobalt atoms and the adsorption energies are therefore comparable. The Co-O distances, similarly to the adsorption energies, differ by less than $0.02 \AA$. One sub-surface oxidation site was found, Fig. S4, SI, but, as it was the case for the other surfaces, with an adsorption energy of $-1.39 \mathrm{eV}$ it is not as exothermic as on-surface adsorption.

In the case of the $(11 \overline{2} 0)$ and (112) surfaces, the most favourable adsorption sites are short bridge 2 and short bridge, respectively. Considering previous surfaces, the situation on the $(11 \overline{2} 0)$ and $(11 \overline{2} 1)$ surfaces is different for two reasons: first, the $z$ coordinate of the elevated Co rows differs by less than $0.15 \AA$ which is significantly lower than for the other surfaces. Second, the closest cobalt atoms are 4.05 and $4.23 \AA$

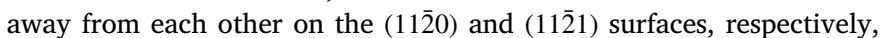
which is $\sim 1.50 \AA$ further away compared to the rest of the surfaces. As a result, bridge sites resemble hollow which therefore loses its predominance and can even become less stable than certain bridge positions. Dependence of Co-O distances on the oxygen coordination number at the adsorption sites is still important as for the other surfaces. Surfaces $(11 \overline{2} 0)$ and $(11 \overline{2} 1)$ were found to be too open to accommodate an oxygen atom in-between the layers.

Generally, on-surface adsorption is more than twice as favourable compared to sub-surface adsorption for all hcp Co surfaces. The most favourable positions are threefold sites, since they offer the highest coordination number where oxygen atom can still remain perfectly centred with respect to the closest equivalent cobalt atoms. The sites presenting less local symmetry (top and long bridge) are the least stable, with differences in the adsorption energies being as large as $1.65 \mathrm{eV}$ between the top and hollow sites on the (0110) surface. As such, the adsorbed oxygen atom tends to occupy the exact positions which would be taken by cobalt atoms in the next layer and adsorbate atoms thus continue the substrate's stacking sequence whenever possible. This tendency helps to explain the finding that only minor structural changes are observed in the positions of slab atoms with respect to their starting geometry.

Comparison with previous theoretical studies is limited owing to the absence of data in the literature on all surfaces except the (0001) surface. The energetic and structural parameters obtained in this work for the (0001) surface are overall in a good agreement with existing data, as shown in Table 4.

\subsubsection{Effect of surface coverage}

Higher coverages (expressed as $N_{\text {Oadsorbed }} / N_{\text {Coperlayer }}$ with a full monolayer, $1.00 \mathrm{ML}$, reached for $N_{\mathrm{O} \text { adsorbed }}=N_{\text {Co per layer }}$ ) have been investigated in order to understand the influence of the concentration of oxygen on the properties of the cobalt surfaces, especially magnetisation.

With a growing coverage of oxygen atoms, the number of possible combinations of their arrangements increases drastically. Although all sites should be considered, the adsorption energies from the single oxygen atom adsorption have been taken as criteria for choosing a reasonable number of site combinations. Additionally, both sub-surface and a combination of on- and sub-surface adsorption were modelled. Included sites and final energetic and structural parameters are listed in Tables S2 and S3, SI.

Energetic and structural parameters: On the (0001) surface, multiple adsorption of oxygen was considered for both threefold fcc and hcp hollow sites along with their combinations. The differences in the cumulative adsorption energies between hollows fcc and hcp decrease with the increase in coverage, showing that the site preference of oxygen becomes negligible. Similarly to the adsorption of one oxygen atom, the adsorbed oxygen atoms situated closer to the surface have the lowest adsorption energies. Additionally, the distance between the oxygen atoms increases after relaxation and displacement towards one edge of the triangular hollow facet can be observed owing to the lateral interactions between oxygen atoms which are uniformly repulsive for all surfaces. The repulsive interaction is emphasised particularly between the oxygen atoms that are bonded to the same surface cobalt atom and/or are closer to each other. As the repulsion gets stronger with the increase in the number of oxygen atoms, the adsorbates are moving further away from the surface. Generally, oxygen co-adsorption induces similar relaxation of the lattice and layer displacements as single oxygen adsorption but modified by the mutual effect of oxygen atoms on interposed cobalt atoms. With full coverage reached, small expansion in the interlayer distance between the second and third cobalt layer can be observed, emphasizing the stronger impact of multiple $\mathrm{O}$ atoms on the second layer compared to the single oxygen adsorption.

The cumulative adsorption energy becomes more positive with the increase in oxygen coverage, Table S2, SI, a trend which has already been reported for other transition metals [58,77] and the Co (0001) surface [39]. However, for the rest of the hcp surfaces, the cumulative adsorption energy is lowered upon the adsorption of two oxygen atoms before becoming less negative with a further increase in the number of oxygen atoms. This unusual trend is connected to the $\mathrm{Co}-\mathrm{O}$ island forma- 


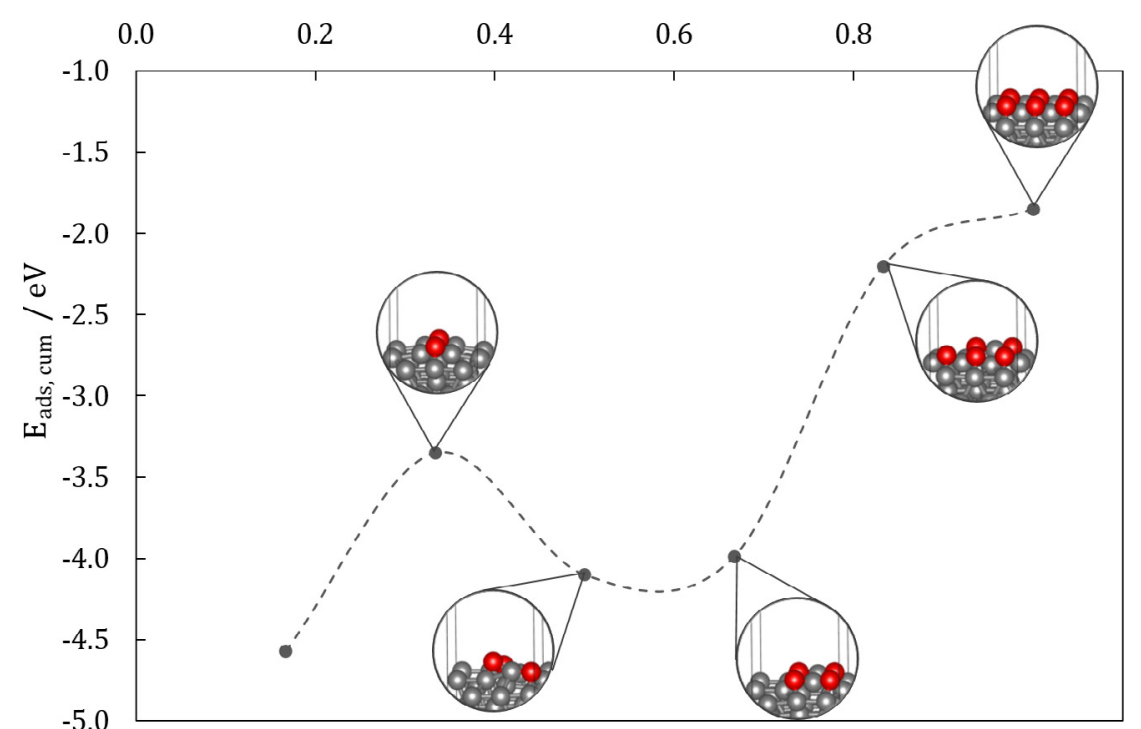

Fig. 3. Calculated cumulative adsorption energies as a function of the oxygen coverage for the $(01 \overline{1} 0)$ surface. tion, as shown in Fig. 3 for the $(01 \overline{1} 0)$ surface. The cobalt atom within the $\mathrm{Co}-\mathrm{O}$ island reduces to some extent the repulsive forces between oxygen atoms, thereby lowering the cumulative adsorption energy. Moreover, the distance between the oxygen atoms and the surface is shortened. With coverages higher than $0.40-0.50 \mathrm{ML}$, the influence of the cobalt atom in the $\mathrm{Co}-\mathrm{O}$ islands decreases while the oxygen-oxygen repulsion becomes dominant, making the cumulative adsorption energy more and more positive with each added oxygen. Correspondingly, the oxygen atoms adsorb slightly further away from the surface. This trend can also be observed on the (0001) surface, where the adsorption of three and four oxygen atoms ( 0.33 and $0.44 \mathrm{ML}$, respectively) results in similar cumulative adsorption energies. In the case of four oxygen atoms, the cobalt atom in the $\mathrm{Co}-\mathrm{O}$ island is placed in the centre of three oxygen atoms, leaving repulsion with the fourth oxygen atom unhindered and the cumulative adsorption energy therefore does not experience as major changes as for the other surfaces. The existence of the cobalt-oxygen islands has already been confirmed experimentally on different hcp Co surfaces [78-80].

Results for higher coverages of sub-surface adsorption and combined on- and sub-surface adsorption are summarised in Table S3, SI, for the surfaces that showed potential to accommodate a single oxygen atom in between the first and second layer of the slab. For all coverages up to $1.00 \mathrm{ML}$, the (0001) surface showed no preference for complete sub-surface oxidation, or on-surface oxidation with one oxygen atom positioned under the surface. Moreover, after geometry optimisation, mixed on- and sub-surface adsorption often resulted in a structure with sub-surface oxygen atoms above the surface. Any higher coverage subsurface adsorption on the (1011) surface resulted in at least one of the oxygen atoms relaxing on top of the surface with considerable disruptions of the metallic slab; the same applies to all on- and sub-surface combinations. The only systems that showed any propensity towards mixed on- and sub-surface adsorption are $\sim 0.70$ and $1.00 \mathrm{ML}$ of oxygen on the $(01 \overline{1} 0)$ surface, with one oxygen atom located in the sub-surface position and the rest in the hollow on-surface positions. Surprisingly, systems with uneven numbers of oxygen atoms per unit cell experienced high levels of deformation. This behaviour could be assigned to the efforts of on-surface oxygen atoms to bind with the surface cobalt atom that became elevated by the oxygen atom placed under the surface. In view of the above, mixed oxidation has only been included in the thermodynamic analysis of the (0110) surface.

Available experimental data mainly consist of structural parameters. For example, for the $(01 \overline{1} 0)$ surface and a high coverage of three-fold chemisorbed oxygen, low energy electron diffraction (LEED) analysis gave values of $0.74 \pm 0.05 \AA$ for oxygen-surface distance, $1.13 \pm 0.10 \AA$ for oxygen-cobalt lateral distances, and $1.83 \pm 0.10 \AA$ for oxygen-cobalt bond distances, with expansion of the first inter-layer spacing to $0.90 \AA$ [81]. For the full coverage in the hollow position in this work, GGA+U gives $0.764 \AA, 1.079 \AA$, $1.801 \AA$ for the oxygen-surface, oxygen-cobalt lateral, and oxygen-cobalt bond distances, respectively, with the first inter-layer spacing expanded to $0.85 \AA$. All distances obtained in this study are within the experimental ranges, thus justifying the chosen model and providing assurance that the predicted structures are reliable.

Work function and magnetisation: Hybridisation of the electronic bands of the oxygen with the bands of the substrate cobalt atoms leads to significant changes in the electronic properties of the topmost layers. Relative to the clean surface, the work function increases with the number of adsorbed oxygen atoms, Table S4, SI. This behaviour is characteristic for the adsorption of oxygen on any metal due to a significant transfer of electrons from the surface atoms to the oxygen (resulting from differences in the electronegativity: $\chi_{\mathrm{O}}=3.44 \mathrm{eV}$ and $\chi_{\mathrm{Co}}=1.88 \mathrm{eV}[82]$ ) generating a large inward pointing surface dipole moment. The changes in the work function of the fully covered and clean surfaces are between 1.40 and $1.90 \mathrm{eV}$, depending on the surface (except for $(11 \overline{2} 1$ ) where the difference is $0.47 \mathrm{eV}$ ). This corresponds well to previous experimental results [83] and similar measurements carried out on other transition metals, where metals with electronegativity closer to oxygen do not experience changes as big as cobalt $\left(\mathrm{Rh} \sim 1.50 \mathrm{eV}\right.$ [84] ( $\left.\chi_{\mathrm{Rh}}=2.28\right), \mathrm{Pd} \sim 1.60 \mathrm{eV}[85]\left(\chi_{\mathrm{Pd}}=2.20\right)$, $\mathrm{Ru} \sim 1.61 \mathrm{eV}$ [77] $\left.\left(\chi_{\mathrm{Ru}}=2.20\right), \mathrm{Pb} \sim 0.84 \mathrm{eV}[86]\left(\chi_{\mathrm{Pb}}=2.33\right)\right)$. The (112̄1) surface is an exception as the first layer cobalt atoms are located far away from each other ( $4.23 \AA$ ), which results in longer Co-O distances and decreased charge transfer.

The magnetisation trend for all surfaces is shown in Fig. 4 and values are listed in Table S4, SI. The surface atoms have larger magnetic moments than the bulk atoms (magnetic moment higher from $0.08 \mu_{\mathrm{B}}$ for the (0001), to $0.37 \mu_{\mathrm{B}}$ for the (112̄1) surface), which is influenced by the narrowing of the $3 \mathrm{~d}$ electron bands. When oxygen adsorption takes place, an initial enhancement of the magnetic moment is the result of the oxygen-induced surface expansion with a drop of magnetisation noted only when the oxide is formed and/or oxygen penetrates into the metal slab. Bigger changes can initially be triggered by the formation of $\mathrm{Co}-\mathrm{O}$ islands. As the oxygen is adsorbed and the coverage is growing, magnetisation drops as expected, but only for two surfaces, (0001) and (0110). For a full monolayer of oxygen, cobalt atoms in the first layer of the (0001) surface became almost nonmagnetic with a magnetic moment 


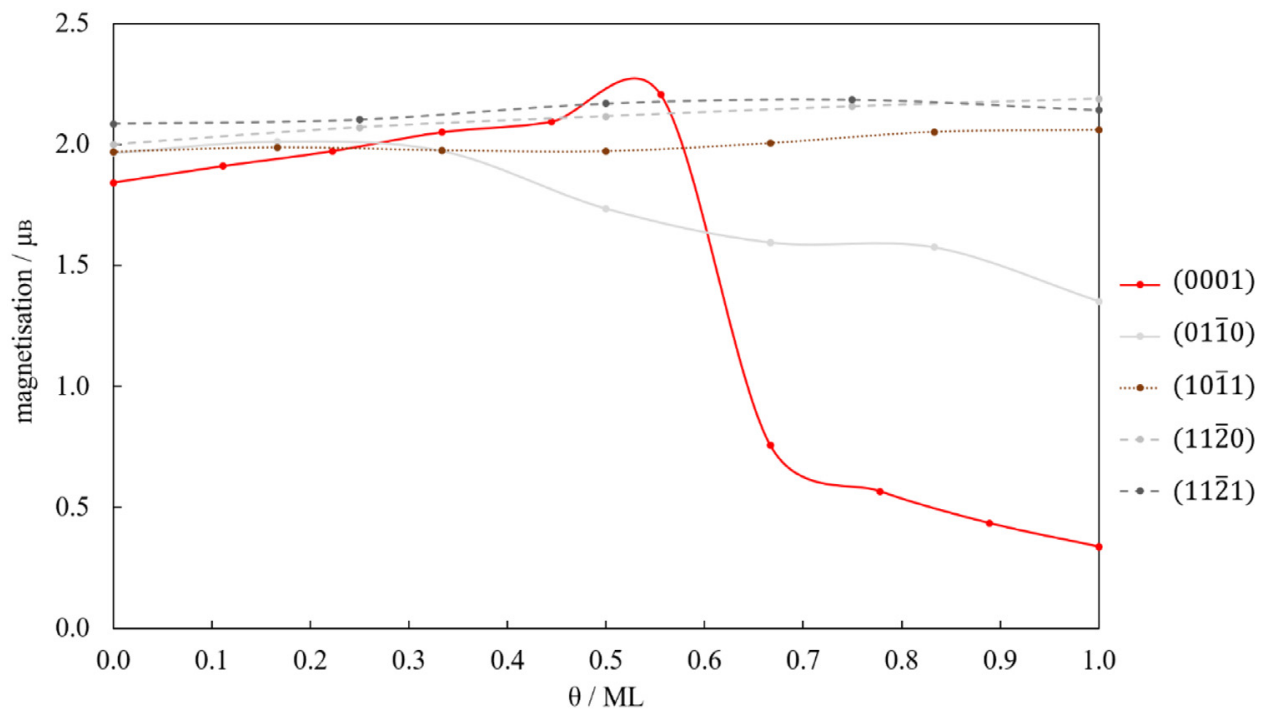

Fig. 4. Magnetisation as a function of the oxygen coverage for five low Miller index hcp Co surfaces.
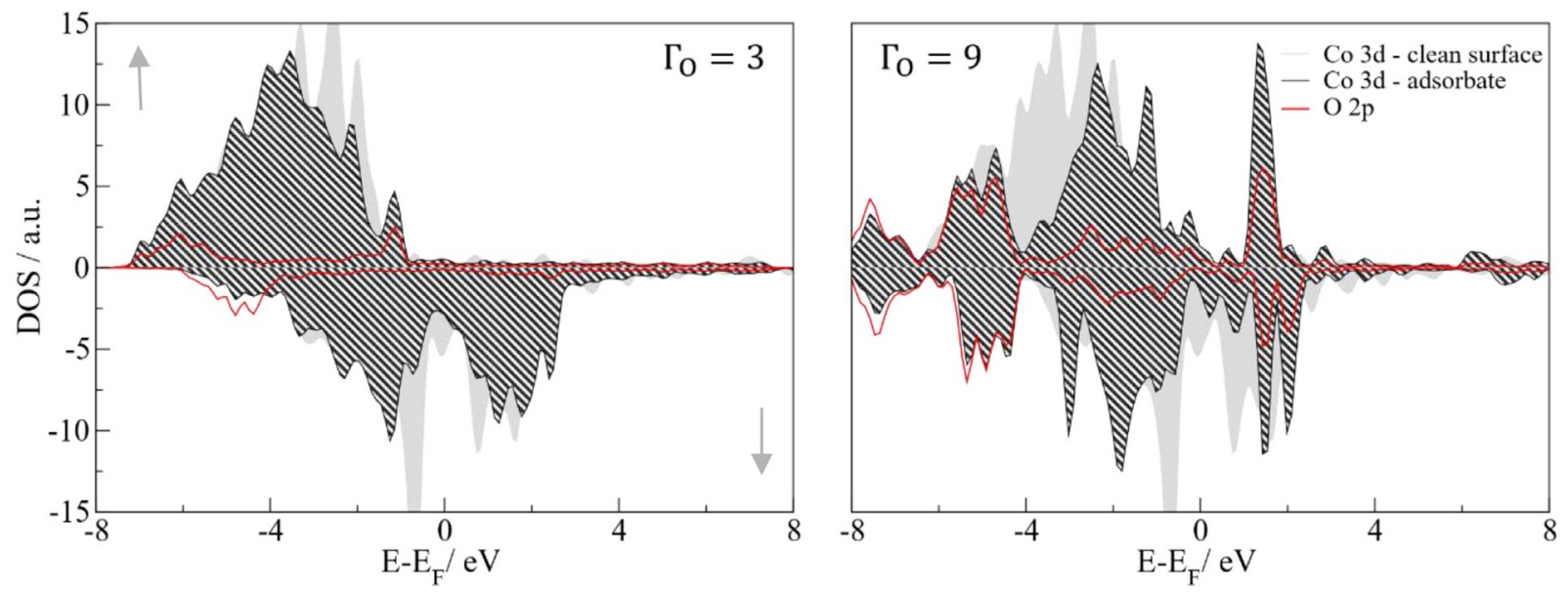

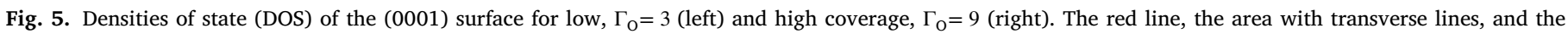

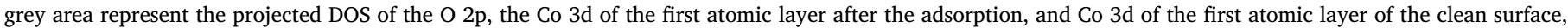

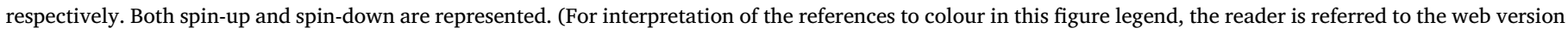
of this article.)

of only $0.34 \mu_{\mathrm{B}}$, while the magnetic moment of the second layer atoms slightly increased by $\sim 0.07 \mu_{\mathrm{B}}$. As magnetisation of the first layer cobalt atoms of the close-packed (0001) surface first rises with increasing the coverage before a steep fall starting from a coverage of about 0.50 ML to the minimal values at $1.00 \mathrm{ML}$, it is possible that higher oxygen coverages are required to observe the same trend for the surfaces that do not experience any lowering of the magnetic moment.

Electronic structure: DOS and d-band centre: For the analysis of the Co$\mathrm{O}$ interactions, densities of states (DOS) have been plotted. Fig. 5 shows the $\mathrm{O} 2 \mathrm{p}$ and Co $3 \mathrm{~d}$ orbitals of the first layer atoms for low and high coverage $\left(\Gamma_{\mathrm{O}}=3\right.$ and $\left.\Gamma_{\mathrm{O}}=9\right)$, along with the Co $3 \mathrm{~d}$ of the first layer atoms of the clean (0001) surface. The DOS for the remaining coverages can be found in Fig. S5, SI. A spin-split of O 2p overlaps with the Co 3d states throughout the whole range considered, with a striking hybridisation peak between oxygen and cobalt occurring at $\sim-5.5 \mathrm{eV}$ for low coverages $\left(\Gamma_{\mathrm{O}}<5\right)$ and splitting into two peaks at $\sim-5.0$ and $\sim-7.5 \mathrm{eV}$ for high coverages $\left(\Gamma_{\mathrm{O}}>6\right)$. This shift of the hybridisation peak and widening of overall $\mathrm{O} 2 \mathrm{p}$ energy distribution with the increase in the number of adsorbed oxygen atoms is caused by the increasing oxygen-oxygen repulsion, in line with the structural parameters discussed above. The majority of the anti-bonding states are located close to the Fermi level, between 1.0 and $2.0 \mathrm{eV}$, for both low and high coverages.
Where the coverage is below $0.50 \mathrm{ML}$, the $\mathrm{O} 2 \mathrm{p}$ bands experience splitting which proves that there are no magnetically inactive layers on the Co surface due to the oxygen adsorption. It is also an indication of the presence of the oxygen magnetic moment, which for three adsorbed oxygen atoms on the (0001) surface equals to $0.28 \mu_{\mathrm{B}}$ per oxygen atom. Following further adsorption, the spin polarisation vanishes, which is represented with a symmetry of up and down spins and can easily be seen in Fig. 5, causing the loss of magnetisation of the first layer cobalt atoms. This is in agreement with the assignment of the formation of $\mathrm{CoO}$ at room temperature, and mainly $\mathrm{Co}_{3} \mathrm{O}_{4}$ at low temperatures, as discussed below, which are both antiferromagnetic materials at $0 \mathrm{~K}$. The same observations are made for the other surfaces, and, although up and down spins do not reach symmetry, the difference between the clean and covered surfaces is clearly visible (Figs. S6-9, SI). It is possible that further increase in the number of oxygen atoms would lead to the loss of magnetism of the first atomic layer.

The plot of the d-band centre in Fig. 6 (up) shows an initial shift of the spin-up states towards negative energies with the increase of oxygen coverage (up to five oxygen atoms in the surface unit cell) whereas with the further increase from moderate to high coverages $\left(\Gamma_{O}>5\right)$ the $d-$ band centre becomes more positive in energy, and vice versa for spindown. 

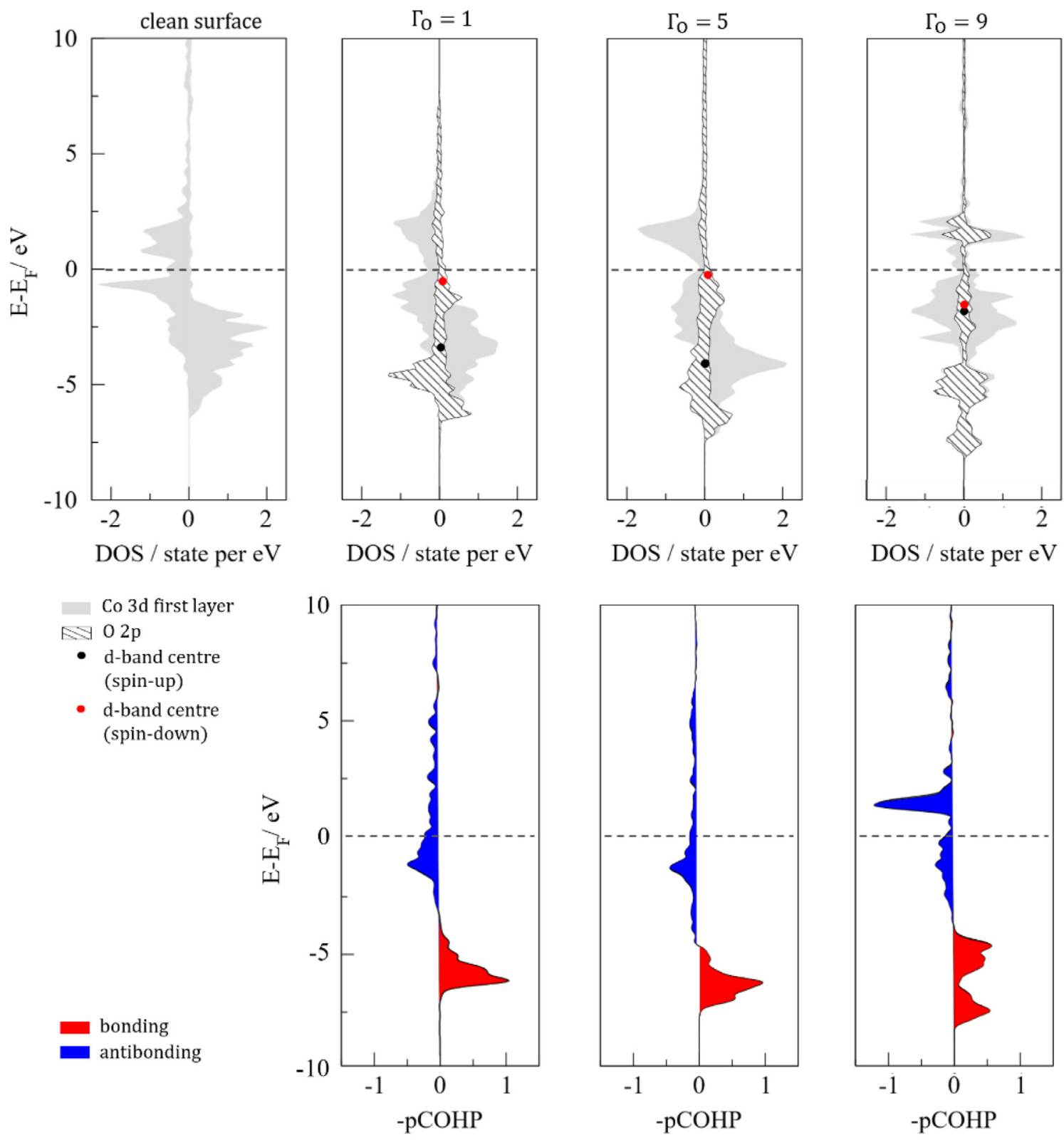

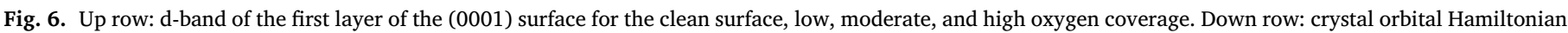
population for the (0001) surface for low, moderate, and high oxygen coverage (pCOHP projections have been made using LOBSTER [88]).

Since there are two contributions to this phenomenon, half coverage represents a switch in dominance between them. The first contribution is interaction of cobalt $d$ electrons with oxygen $2 p$ states, where the latter must become orthogonal with respect to the cobalt atom's unpaired electrons when they come into contact, giving a rise to the kinetic energy due to the repulsive forces. The second contribution originates from the emptying of the antibonding cobalt $d$ states. To prove that with higher coverages second contribution predominates, crystal orbital Hamiltonian populations (COHP) have also been plotted in Fig. 6 (down) for low $\left(\Gamma_{O}=1\right)$, moderate $\left(\Gamma_{O}=5\right)$, and high oxygen coverage $\left(\Gamma_{O}=9\right)$ for the (0001) surface, with bonding and antibonding states represented in red and blue, respectively. While almost no change can be observed in the antibonding populations when going from 1 to 5 adsorbed oxygen atoms, when full coverage is reached, empty antibonding states appear at $\sim 1.5 \mathrm{eV}$ above the Fermi level.

Charge analysis: Confirmation of the increase in the work function comes from the Bader analysis [87], Table S4, SI, where it can be seen that oxygen atoms are negatively charged, which implies a charge transfer from the surface to the adsorbate. At low coverages $(\theta<0.50 \mathrm{ML})$, the Bader charge of oxygen on the (0001) surface is close to $1.00 e^{-}$per oxygen atom, which is reduced to $0.69 e^{-}$at $\theta=1.00 \mathrm{ML}$, indicating once again the repulsive force between oxygen atoms and return of some negative charge to the surface. The same trend is followed in all other surfaces, with the exception of the first structure experiencing $\mathrm{Co}-\mathrm{O}$ island formation, where both Bader charges and the work function complement the adsorption energies.

Moreover, charge rearrangements leading to the bond dipole were analysed through the charge density difference as the difference between the charge density of a system containing oxygen adsorbed on the surface and the sum of the charge densities of the two subsystems, namely the freestanding adsorbate layer and the slab in the same geometry, $\Delta \rho=\rho_{\text {surface+oxygen }}-\left(\rho_{\text {surface }}+\rho_{\text {oxygen }}\right)$. Fig. 7 shows the charge density difference as an isosurface and the plane-averaged differential charge distribution along the $z$ axis for one oxygen atom adsorbed on the 

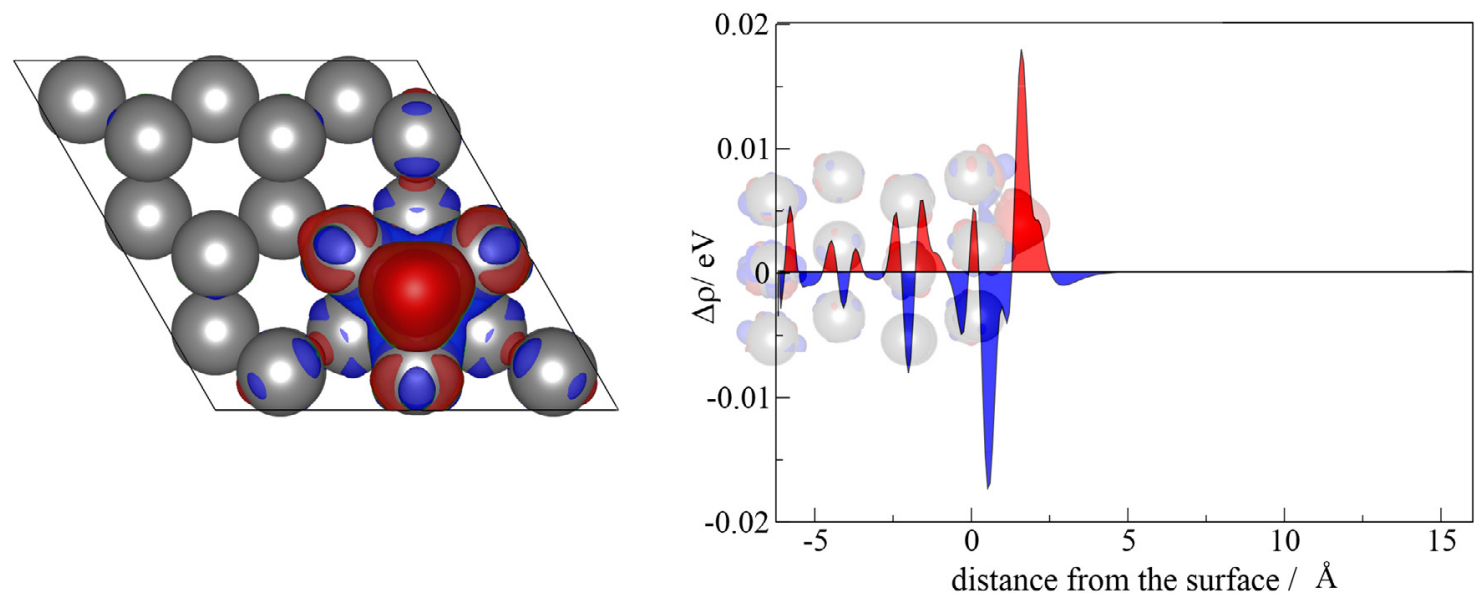

Fig. 7. Charge density difference plotted as an isosurface (left) and as $x, y$ plane-average (right) for single oxygen adsorbed on the (0001) surface. Red and blue colours represent charge accumulation and depletion, respectively (isosurface value $=0.0062$ electrons per $\AA^{3}$ ). (For interpretation of the references to colour in this figure legend, the reader is referred to the web version of this article.)
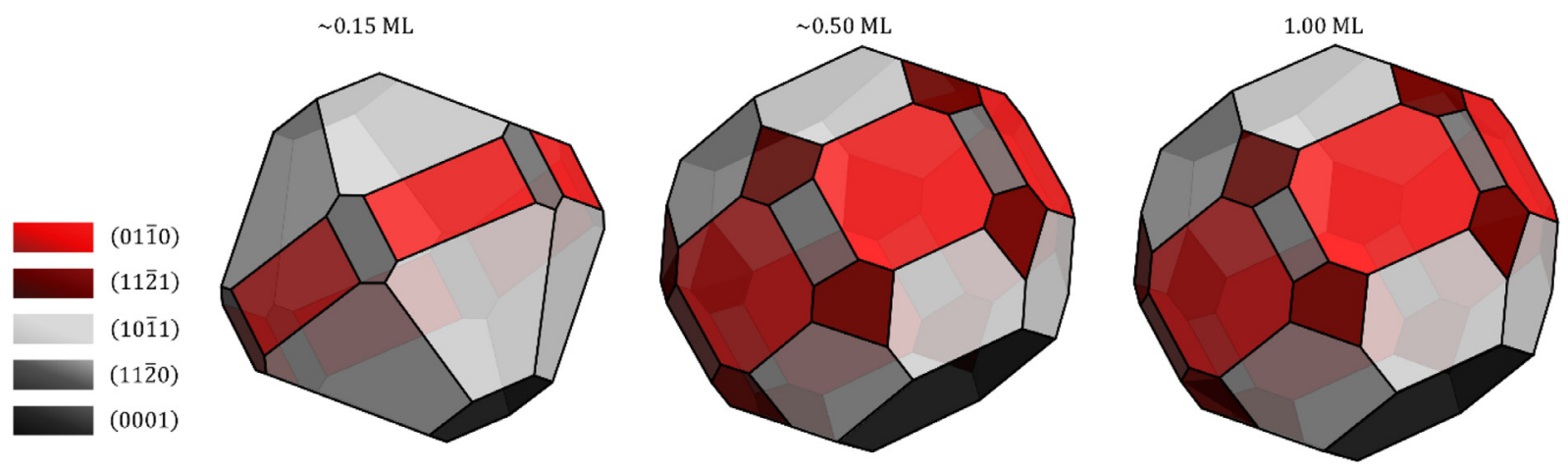

Fig. 8. Wulff crystal morphologies for hep Co nanoparticles with $\sim 0.15, \sim 0.50$, and 1.00 ML of oxygen.

(0001) surface, with red regions denoting charge accumulation and blue regions charge depletion. Redistribution of charge takes place mostly in between the closest surface atoms and adsorbed oxygen, whereas almost no charge transfer occurs further away due to weak interactions between the remote cobalt atoms and the adsorbate. Altogether, both charge accumulation at oxygen and charge depletion at nearby cobalt atoms contribute to the inward-facing electric dipole, which is responsible for the increase of the work function.

Morphology: Fig. 8 displays the Wulff morphologies for low ( $\sim 0.15 \mathrm{ML})$, moderate $(\sim 0.50 \mathrm{ML})$, and full coverages of oxygen. The influence of the oxygen adsorption on the overall structure of the nanoparticles cannot be neglected as major changes happen even with only one adsorbed atom. Going from 0.15 to $0.50 \mathrm{ML}$ triggers a significant increase in the share of the (0001) surface and reappearance of the (112 1$)$ surface with only minor changes in surface ratios when reaching a full oxygen monolayer. The most important result of the transformations from clean to fully covered nanoparticle is the enhancement in the expose areas of the (0001) and $(01 \overline{1} 0)$ surfaces since these planes experience drastic lowering of magnetisation upon oxygen adsorption, which should consequently lead to a significant loss in the total magnetisation of the nanoparticles.

\subsubsection{Phase diagram of surface energy}

Surface phase diagrams have been constructed for all surfaces (Figs. S10-13 SI) for a wide range of chemical potentials, from -6.0 to $0.0 \mathrm{eV}$, and shown for the (0001) surface in Fig. 9. Scale of pressure ratio (with $p_{0}=1$ bar) for $\sim 36-37^{\circ} \mathrm{C}$, as the temperature matching the body envi- ronment, has been provided as well as conditions of the oxygen chemical potential for the formation of the two most stable cobalt oxides, namely cubic rocksalt-structured $\mathrm{CoO}$ at $-2.22 \mathrm{eV}$ and normal spinel $\mathrm{Co}_{3} \mathrm{O}_{4}$ at $-0.45 \mathrm{eV}$. Under these conditions the following reactions occur:

$\mathrm{Co}+1 / 2 \mathrm{O}_{2} \rightleftarrows \mathrm{CoO}$

$3 \mathrm{CoO}+1 / 2 \mathrm{O}_{2} \rightleftarrows \mathrm{Co}_{3} \mathrm{O}_{4}$

From a thermodynamics point of view, the surface or nanoparticle composition in equilibrium with the oxygen environment under particular chosen conditions of temperature and pressure is determined by the minimum energy over possible compositions. As the chemical potential of oxygen becomes less negative, successive surfaces containing higher oxygen coverages will become thermodynamically stable. Therefore, considering the phase diagram of the (0001) surface shown in Fig. 9, the clean surface will appear at negative potentials up to $\sim-3.70 \mathrm{eV}$. Upon further increase of $\Delta \mu_{\mathrm{O}_{2}}$ from -3.70 to $-2.22 \mathrm{eV}$, oxygen overlayers become progressively more favourable than the clean surface, with $\Gamma_{\mathrm{O}}=1$ dominating between -3.70 and $-3.45 \mathrm{eV}, \Gamma_{\mathrm{O}}=2$ between -3.45 and $-3.35 \mathrm{eV}$, where the surface with three adsorbed oxygen atoms becomes dominant. At $\sim-3.00 \mathrm{eV}$ the most stable surface contains four adsorbed oxygen atoms, and then from $\sim-2.70 \mathrm{eV}$ to the boundary where cobalt oxides are formed, a coverage with five $\mathrm{O}$ atoms persists. Around the upper limit of $\Delta \mu_{\mathrm{O}_{2}}=-2.20 \mathrm{eV}$, adsorbed oxygen overlayers may exist as metastable structures, with $\mathrm{CoO}$ representing the thermodynamically most stable phase. Other surfaces follow the same pattern, with the clean surface being stable at low chemical potentials (up 


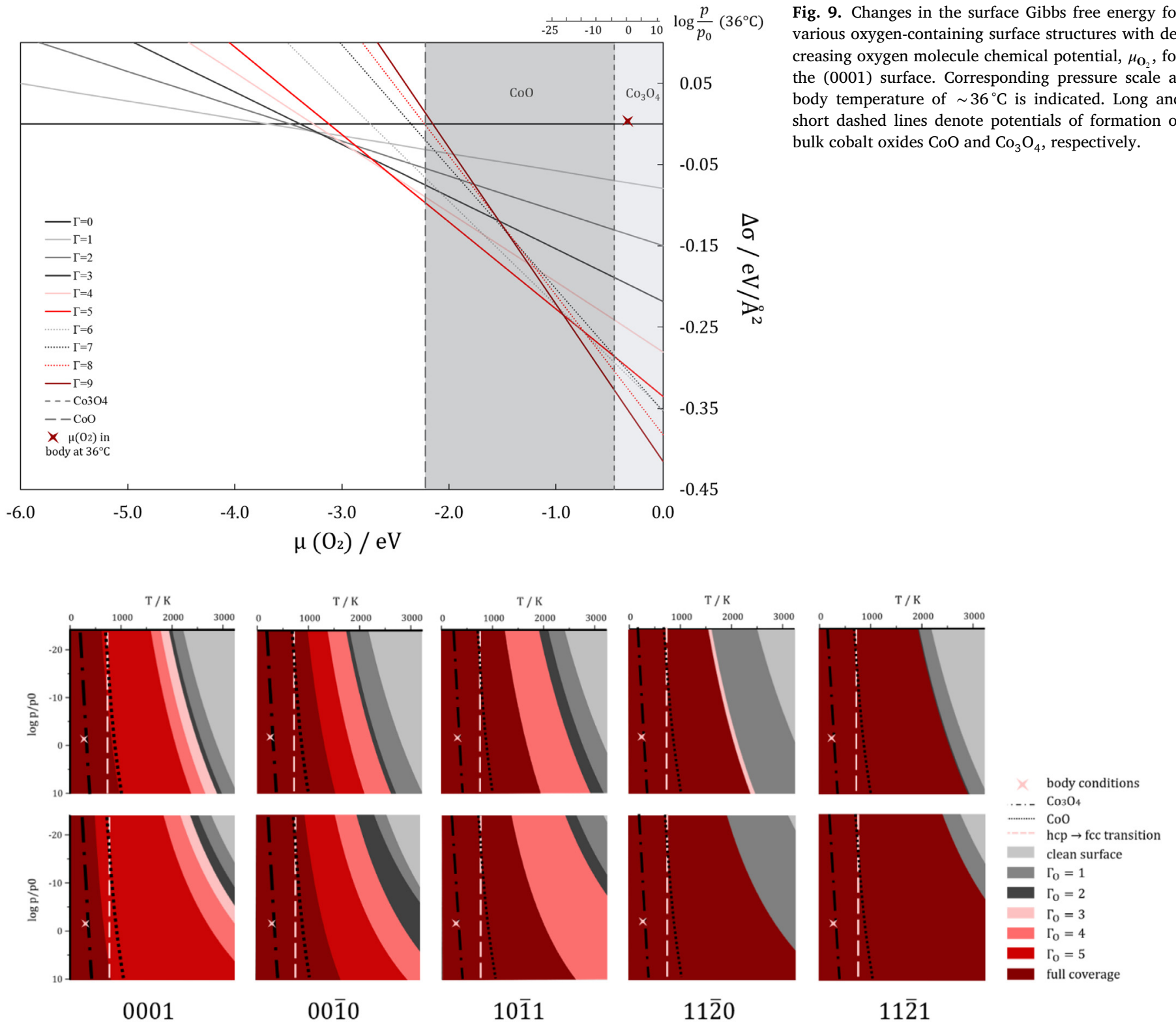

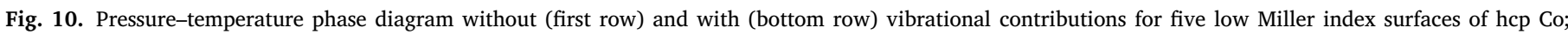
pressure and temperature scales are the same for all figures. Conditions of formation of both cobalt oxides and hcp $\rightarrow$ fcc phase transition have been provided.

to -4.00 or $-3.50 \mathrm{eV}$ ) through surfaces with a few chemisorbed oxygen atoms dominating at moderately negative potentials (from -3.50 to $-2.50 \mathrm{eV}$ ) to high coverages leading to oxides at potentials higher than $-2.22 \mathrm{eV}$.

Fig. 10 shows phase diagrams as a function of pressure and temperature for all the surfaces. Regardless of the pressure, the ordering of the oxygen coverages is kept constant, and the same phase should be observable for different pressures with accordingly adjusted temperature, unless the formation of the structure is constrained by slow kinetics. Inclusion of vibrational corrections showed noticeable effects, with transitions between surfaces with different numbers of adsorbed oxygen atoms shifting down by approximately $100 \mathrm{~K}$. The overall order of the phases does not change, but their ratio does. These results correspond well to the experimentally tested formation of cobalt oxides $[89,90]$ and temperatures obtained for the $\mathrm{Co}_{3} \mathrm{O}_{4} \rightarrow \mathrm{CoO}$ conversion. For the (0001) surface, spectroscopic methods revealed that high oxygen exposure ( $p_{\mathrm{O}_{2}}=0.5$ Torr) at $295 \mathrm{~K}$ tends to form the spinel oxide, but the situation changes with annealing at $700 \mathrm{~K}$ in favour of $\mathrm{CoO}$; at low exposures $\mathrm{CoO}$ is formed even at $295 \mathrm{~K}$ [90]. Meanwhile, for the
(1120) surface, conversion happens at $170-230 \mathrm{~K}$ with $\mathrm{CoO}$ intensities growing until $450 \mathrm{~K}$ [83]. These examples and other data [78,91] correspond well with obtained theoretical phase diagrams.

If the partial pressure of oxygen in blood is considered ( $p=0.133 \mathrm{bar}$ ) at body temperature $\left(\sim 36-37^{\circ} \mathrm{C}\right)$, it can easily be seen that, with a corresponding oxygen chemical potential of $-0.31 \mathrm{eV}$, the predominant structure is far into the cobalt oxides area, making it impossible to obtain or retain a clean surface under these conditions.

\section{Conclusions}

To test the influence of oxygen on hcp cobalt nanoparticles in vivo, adsorption on low Miller index surfaces has been investigated by means of periodic DFT calculations. The results indicate spontaneous adsorption with oxygen's preference for the sites with the highest coordination number and to remain centred in between structurally equivalent surface atoms for all surfaces. The coverage-dependent energetic and structural parameters consistently show the dipole nature of the oxygencobalt bond. When considering thermodynamics, for temperatures be- 
low the hcp $\rightarrow$ fcc transition a full monolayer of oxygen is thermodynamically preferred over a whole range of relevant pressures.

From these findings, aforementioned concerns can be appropriately discussed:

1) If only body conditions are observed $\left(\sim 36-37^{\circ} \mathrm{C}\right.$ and $p_{\mathrm{O}_{2}}=$ 0.133 bar), $\mathrm{Co}_{3} \mathrm{O}_{4}$ represents the thermodynamically most stable phase. Consequently, surfaces rapidly lose their magnetisation with the increase in the chemical potential of oxygen. Thus, if cobalt nanoparticles are injected in the blood system, non-magnetic oxide would be formed, making re-establishing of highly needed magnetisation by far more important issue than toxicity itself.

2) To prevent contact between surfaces and oxygen, a covering layer that would provide biocompatibility without affecting the magnetic properties of cobalt should be added. Additional functionalisation possibilities such as drug delivery, although not crucial, are also of significant importance.

Future work will focus on finding the most appropriate coating and making it possible to utilise the promising properties cobalt nanoparticles are offering in respect with the further improvement of cancer treatments.

\section{Declaration of interest}

The authors declare that they have no known competing financial interests or personal relationships that could have appeared to influence the work reported in this paper.

\section{Acknowledgements}

BF thanks Cardiff University for support through a Research Scholarship from the School of Chemistry. We acknowledge the Engineering and Physical Sciences Research Council (Grant nos. EP/R512503/1, $\mathrm{EP} / \mathrm{K} 016288 / 1$, and EP/K009567/2) for funding. This research was undertaken using the Supercomputing Facilities at Cardiff University operated by ARCCA on behalf of the Cardiff Supercomputing Facility and the HPC Wales and Supercomputing Wales (SCW) projects. We acknowledge the support of the latter, which is part-funded by the European Regional Development Fund (ERDF) via Welsh Government. Via our membership of the UK's HPC Materials Chemistry Consortium, which is funded by EPSRC (EP/L000202), this work made use of the facilities of ARCHER, the UK's national high-performance computing service, which is funded by the Office of Science and Technology through EPSRC's High End Computing Programme. Information on the data underpinning the presented results, including how to access them, can be found in the Cardiff University Research Portal at http://doi.org/10.17035/d.2018.0052762507.

\section{Supplementary materials}

Supplementary material associated with this article can be found, in the online version, at doi:10.1016/j.mtla.2019.100381.

\section{References}

[1] World Health Assembly, Cancer prevention and control in the context of an integrated approach, in: Resolution WHA70.12 adopted by the seventieth World Health Assembly, 22, 2017, pp. 1-9.

[2] Y. Chen, P. Jungsuwadee, M. Vore, D.A. Butterfield, D.K. St. Clair, Collateral damage in cancer chemotherapy: oxidative stress in nontargeted tissues, Mol. Interv. 7 (2007) 147-156, doi:10.1124/mi.7.3.6.

[3] S. Mitragotri, D.G. Anderson, X. Chen, E.K. Chow, D. Ho, A.V. Kabanov, J.M. Karp, K. Kataoka, C.A. Mirkin, S.H. Petrosko, J. Shi, M.M. Stevens, S. Sun, S. Teoh, S.S. Venkatraman, Y. Xia, S. Wang, Z. Gu, C. Xu, Accelerating the translation of nanomaterials in biomedicine, ACS Nano 9 (2015) 6644-6654, doi:10.1021/acsnano.5b03569.

[4] A.J. Cole, V.C. Yang, A.E. David, Cancer theranostics: the rise of targeted magnetic nanoparticles, Trends Biotechnol. 29 (2011) 323-332, doi:10.1016/j.tibtech.2011.03.001.
[5] J. Beik, Z. Abed, F.S. Ghoreishi, S. Hosseini-Nami, S. Mehrzadi, A. Shakeri-Zadeh, S.K. Kamrava, Nanotechnology in hyperthermia cancer therapy: from fundamental principles to advanced applications, J. Control. Release 235 (2016) 205-221, doi:10.1016/j.jconrel.2016.05.062.

[6] P.B. Santhosh, N.P. Ulrih, Multifunctional superparamagnetic iron oxide nanoparticles: promising tools in cancer theranostics, Cancer Lett. 336 (2013) 8-17, doi:10.1016/j.canlet.2013.04.032.

[7] A.H. Habib, C.L. Ondeck, P. Chaudhary, M.R. Bockstaller, M.E. Mchenry, Evaluation of iron-cobalt/ferrite core-shell nanoparticles for cancer thermotherapy evaluation of iron-cobalt/ferrite core-shell nanoparticles for cancer thermotherapy, J. Appl. Phys. 103 (2008), doi:10.1063/1.2830975.

[8] J.T. Jang, H. Nah, J.H. Lee, S.H. Moon, M.G. Kim, J. Cheon, Critical enhancements of MRI contrast and hyperthermic effects by dopant-controlled magnetic nanoparticles, Angew. Chem. - Int. Ed. 48 (2009) 1234-1238, doi:10.1002/anie.200805149.

[9] S. Moise, E. Céspedes, D. Soukup, J.M. Byrne, A.J. El Haj, N.D. Telling, The cellular magnetic response and biocompatibility of biogenic zinc- and cobalt-doped magnetite nanoparticles, Sci. Rep. 7 (2017) 1-11. https://doi.org/10.1038/srep39922.

[10] L.V. Lutsev, A.I. Stognij, N.N. Novitskii, Giant magnetoresistance in semiconductor/granular film heterostructures with cobalt nanoparticles, Phys. Rev. B - Condens. Matter Mater. Phys. 80 (2009) 40-42, doi:10.1103/PhysRevB.80.184423.

[11] M. Trépanier, A.K. Dalai, N. Abatzoglou, Synthesis of CNT-supported cobalt nanoparticle catalysts using a microemulsion technique: role of nanoparticle size on reducibility, activity and selectivity in fischer-tropsch reactions, Appl. Catal. A Gen. 374 (2010) 79-86, doi:10.1016/j.apcata.2009.11.029.

[12] L.O. Simonsen, H. Harbak, P. Bennekou, Cobalt metabolism and toxicology-A brief update, Sci. Total Environ. 432 (2012) 210-215, doi:10.1016/j.scitotenv.2012.06.009.

[13] Y. Liu, H. Zhu, H. Hong, W. Wang, F. Liu, Can zinc protect cells from the cytotoxic effects of cobalt ions and nanoparticles derived from metal-on-metal joint arthroplasties? Bone Jt. Res. 6 (2017) 649-655, doi:10.1302/2046-3758.612.BJR-2016-0137.R2.

[14] S.C. Petitto, E.M. Marsh, G.A. Carson, M.A. Langell, Cobalt oxide surface chemistry: the interaction of $\mathrm{CoO}(100), \mathrm{Co}_{3} \mathrm{O}_{4}(110)$ and $\mathrm{Co}_{3} \mathrm{O}_{4}(111)$ with oxygen and water, J. Mol. Catal. A Chem. 281 (2008) 49-58, doi:10.1016/j.molcata.2007.08.023.

[15] H.A. Engelhardt, D. Menzel, Adsorption of oxygen on silver single crystal surfaces, Surf. Sci. 57 (1976) 591-618, doi:10.1016/0039-6028(76)90350-2.

[16] P. Michel, C. Jardin, Oxygen adsorption and oxide formation on $\operatorname{Cr}(100)$ and $\operatorname{Cr}(110)$ surfaces, Surf. Sci. 36 (1973) 478-487, doi:10.1016/0039-6028(73)90396-8.

[17] F. Besenbacher, J.K. Nørskov, Oxygen chemisorption on metal surfaces: general trends for $\mathrm{Cu}, \mathrm{Ni}$ and $\mathrm{Ag}$, Prog. Surf. Sci. 44 (1993) 5-66, doi:10.1016/0079-6816(93)90006-H.

[18] H. Zhang, A. Soon, B. Delley, C. Stampfl, Stability, structure, and electronic properties of chemisorbed oxygen and thin surface oxides on $\operatorname{Ir}(111)$, Phys. Rev. B 78 (2008) 045436, doi:10.1103/PhysRevB.78.045436.

[19] R.B. Getman, Y. Xu, W.F. Schneider, Thermodynamics of environment-dependent oxygen chemisorption on Pt(111), J. Phys. Chem. C 112 (2008) 9559-9572, doi:10.1021/jp800905a

[20] H. Shi, C. Stampfl, First-principles investigations of the structure and stability of oxygen adsorption and surface oxide formation at Au(111), Phys. Rev. B 76 (2007) 075327, doi:10.1103/PhysRevB.76.075327.

[21] S. López-Moreno, A.H. Romero, Atomic and molecular oxygen adsorbed on (111) transition metal surfaces: $\mathrm{Cu}$ and Ni, J. Chem. Phys. 142 (2015) 154702, doi:10.1063/1.4917259

[22] J.K. Gimzewski, S. Affrossman, M.T. Gibson, L.M. Watson, D.J. Fabian, Oxidation of scandium by oxygen and water studied by XPS, Surf. Sci. 80 (1979) 298-305, doi:10.1016/0039-6028(79)90690-3.

[23] J. Wang, Y. Wang, G. Wu, X. Zhang, X. Zhao, M. Yang, Ab initio study of the structure and magnetism of atomic oxygen adsorbed $\operatorname{sen}(n=2-14)$ clusters, Phys. Chem. Chem. Phys. 11 (2009) 5980, doi:10.1039/b902627d.

[24] Y. Zhu, Y. Sun, A study of the oxygen adsorption and initial oxidation on polycrystalline zinc by AES line shapes and EELS, Surf. Sci. 275 (1992) 357-364, doi:10.1016/0039-6028(92)90808-J.

[25] R. Nakamura, J.G. Lee, D. Tokozakura, H. Mori, H. Nakajima, Formation of hollow ZnO through low-temperature oxidation of Zn nanoparticles, Mater. Lett. 61 (2007) 1060-1063, doi:10.1016/j.matlet.2006.06.039.

[26] G. Strasser, G. Rosina, E. Bertel, P.P. Netzer, Surface oxidation of cerium and lanthanum, Surf. Sci. 152-153 (1985) 765-775, doi:10.1016/0039-6028(85)90486-8.

[27] M.S. Palmer, M. Neurock, M.M. Olken, Periodic density functional theory study of the dissociative adsorption of molecular oxygen over $\mathrm{La}_{2} \mathrm{O}_{3}$, J. Phys. Chem. B 106 (2002) 6543-6547, doi:10.1021/jp020492x.

[28] A.G. Ritchie, A review of the rates of reaction of uranium with oxygen and water vapour at temperatures up to $300^{\circ} \mathrm{C}$, J. Nucl. Mater. 102 (1981) 170-182, doi:10.1016/0022-3115(81)90557-2.

[29] M.N. Huda, A.K. Ray, Density functional study of $\mathrm{O}_{2}$ adsorption on (100) surface of $\gamma$-uranium, Int. J. Quantum Chem. 102 (2005) 98-105. http://dx.doi.org/10.1002/qua.20365.

[30] B.K. Min, X. Deng, D. Pinnaduwage, R. Schalek, C.M. Friend, Oxygen-induced restructuring with release of gold atoms from Au(111), Phys. Rev. B - Condens. Matter Mater. Phys. 72 (2005) 1-4, doi:10.1103/PhysRevB.72.121410.

[31] A. Michaelides, K. Reuter, M. Scheffler, When seeing is not believing: oxygen on $\mathrm{Ag}(111)$, a simple adsorption system? J. Vac. Sci. Technol. A Vac., Surf., Film 23 (2005) 1487-1497, doi:10.1116/1.2049302

[32] H. Tang, A. Van Der Ven, B.L. Trout, Phase diagram of oxygen adsorbed on platinum (111) by first-principles investigation, Phys. Rev. B - Condens. Matter Mater. Phys. 70 (2004) 1-10, doi:10.1103/PhysRevB.70.045420. 
[33] V.I. Bukhtiyarov, M. Hävecker, V.V. Kaichev, A. Knop-Gericke, R.W. Mayer, R. Schlögl, Atomic oxygen species on silver: photoelectron spectroscopy and x-ray absorption studies, Phys. Rev. B 67 (2003) 235422, doi:10.1103/PhysRevB.67.235422.

[34] J. Gottfried, K. Schmidt, S.L. Schroeder, K. Christmann, Oxygen chemisorption on $\mathrm{Au}(110)-(1 \times 2)$ I. Thermal desorption measurements, Surf. Sci. 525 (2003) 184-196, doi:10.1016/S0039-6028(02)02560-8.

[35] G.W. Simmons, Y.N. Wang, J. Marcos, K. Klier, Oxygen adsorption on palladium(100) surface: phase transformations and surface reconstruction, J. Phys. Chem. 95 (1991) 4522-4528, doi:10.1021/j100164a063.

[36] W. Clemens, E. Vescovo, T. Kachel, C. Carbone, W. Eberhardt, Spin-resolved photoemission study of the reaction of $\mathrm{O}_{2}$ with fcc $\mathrm{Co}(100)$, Phys. Rev. B 46 (1992) 4198-4204, doi:10.1103/PhysRevB.46.4198.

[37] S.H. Ma, Z.Y. Jiao, T.X. Wang, X.Q. Dai, Ab initio study on the adsorption of oxygen on Co(111) and its subsurface incorporation, Eur. Phys. J. B 88 (2015) 4, doi:10.1140/epjb/e2014-50292-0.

[38] W. Liu, N. Miao, L. Zhu, J. Zhou, Z. Sun, Adsorption and diffusion of hydrogen and oxygen in FCC-Co: a first-principles study, Phys. Chem. Chem. Phys. 19 (2017) 32404-32411, doi:10.1039/C7CP07208B.

[39] S.H. Ma, Z.Y. Jiao, T.X. Wang, X.Q. Dai, First-principles studies of oxygen chemisorption on Co(0001), Surf. Sci. 619 (2014) 90-97, doi:10.1016/j.susc.2013.09.015.

[40] A.C. (Ali C. Kizilkaya, J.W. (Hans) Niemantsverdriet, C.J. (Kees-J. Weststrate, Oxygen adsorption and water formation on Co(0001), J. Phys. Chem. C 120 (2016) 4833-4842, doi:10.1021/acs.jpcc.5b08959.

[41] G. Kresse, J. Furthmüller, Efficiency of ab-initio total energy calculations for metals and semiconductors using a plane-wave basis set, Comput. Mater. Sci. 6 (1996) 1550, doi:10.1016/0927-0256(96)00008-0.

[42] W. Kohn, L.J. Sham, Self-consistent equations including exchange and correlation effects, Phys. Rev. 140 (1965), doi:10.1103/PhysRev.140.A1133.

[43] J.P. Perdew, K. Burke, M. Ernzerhof, Generalized gradient approximation made simple, Phys. Rev. Lett. 77 (1996) 3865-3868, doi:10.1103/PhysRevLett.77.3865.

[44] S. Grimme, S. Ehrlich, L. Goerigk, Effect of the damping function in dispersion corrected density functional theory, J. Comput. Chem. 32 (2011) 1456-1465, doi:10.1002/jcc.21759.

[45] D. Santos-Carballal, A. Roldan, N.Y. Dzade, N.H. de Leeuw, Reactivity of $\mathrm{CO}_{2}$ on the surfaces of magnetite $\left(\mathrm{Fe}_{3} \mathrm{O}_{4}\right)$, greigite $\left(\mathrm{Fe}_{3} \mathrm{~S}_{4}\right)$ and mackinawite $(\mathrm{FeS})$, Philos. Trans. R. Soc. A Math. Phys. Eng. Sci. 376 (2018) 20170065, doi:10.1098/rsta.2017.0065.

[46] G. Kresse, D. Joubert, From ultrasoft pseudopotentials to the projector augmented-wave method, Phys. Rev. B 59 (1999) 11-19. https://doi.org/10.1103/PhysRevB.59.1758.

[47] V.I. Anisimov, F. Aryasetiawan, A.I. Lichtenstein, First-principles calculations of the electronic structure and spectra of strongly correlated systems: the LDA + u method, J. Phys. Condens. Matter 9 (1997) 767-808.

[48] C.S. Wang, B.M. Klein, H. Krakauer, Theory of magnetic and structural ordering in iron, Phys. Rev. Lett. 54 (1985) 1852-1855, doi:10.1103/PhysRevLett.54.1852.

[49] A. Walsh, J.L.F. Da Silva, S.-H. Wei, Theoretical description of carrier mediated magnetism in cobalt doped ZnO, Phys. Rev. Lett. 100 (2008) 256401, doi:10.1103/PhysRevLett.100.256401.

[50] L. Wang, T. Maxisch, G. Ceder, Oxidation energies of transition metal oxides within the GGA+U framework, Phys. Rev. B - Condens. Matter Mater. Phys. 73 (2006) 1-6, doi:10.1103/PhysRevB.73.195107.

[51] A. Jain, G. Hautier, S.P. Ong, C.J. Moore, C.C. Fischer, K.A. Persson, G. Ceder, Formation enthalpies by mixing GGA and GGA + u calculations, Phys. Rev. B - Condens. Matter Mater. Phys. 84 (2011) 1-10, doi:10.1103/PhysRevB.84.045115.

[52] A. Cadi-Essadek, A. Roldán, D. Santos-Carballal, P.E. Ngoepe, N.H. de Leeuw, DFT+U study of the electronic, magnetic, and mechanical properties of $\mathrm{Co}, \mathrm{CoO}$, and $\mathrm{Co}_{3} \mathrm{O}_{4}$, (2019). Unpublished work.

[53] W. Betteridge, The properties of metallic cobalt, Prog. Mater. Sci. 24 (1980) 51-142, doi:10.1016/0079-6425(79)90004-5.

[54] G.W. Watson, E.T. Kelsey, N.H. de Leeuw, D.J. Harris, S.C. Parker, Atomistic simulation of dislocations, surfaces and interfaces in MgO, J. Chem. Soc. Faraday Trans. 92 (1996) 433, doi:10.1039/ft9969200433.

[55] R.V. Zucker, D. Chatain, U. Dahmen, S. Hagege, W.C. Carter, New software tools for the calculation and display of isolated and attached interfacialenergy minimizing particle shapes, J. Mater. Sci. 47 (2012) 8290-8302, doi:10.1007/s10853-012-6739-x.

[56] G. Wulff, XXV. Zur frage der geschwindigkeit des wachsthums und der auflösung der krystallflächen, Z. Für Krist. - Cryst. Mater. 34 (1901), doi:10.1524/zkri.1901.34.1.449.

[57] T.J. Chuang, C.R. Brundle, D.W. Rice, Interpretation of the x-ray photoemission spectra of cobalt oxides and cobalt oxide surfaces, Surf. Sci. 59 (1976) 413-429, doi:10.1016/0039-6028(76)90026-1.

[58] W.-X. Li, C. Stampfl, M. Scheffler, Oxygen adsorption on Ag(111): a densityfunctional theory investigation, Phys. Rev. B 65 (2002) 075407, doi:10.1103/PhysRevB.65.075407.

[59] C. Kittel, Introduction to Solid State Physics, John Wiley \& Sons, Inc, 2010, doi:10.1007/978-3-540-93804-0.

[60] G. Herzberg, K.P. Huber, Molecular spectra and molecular structure, 1979. doi:10.1007/978-1-4757-0961-2.

[61] K. Reuter, M. Scheffler, Composition, structure, and stability of $\mathrm{RuO}_{2}$ (110) as a function of oxygen pressu, Phys. Rev. B 65 (2001) 035406, doi:10.1103/PhysRevB.65.035406.

[62] D. Santos-Carballal, A. Roldan, R. Grau-Crespo, N.H. de Leeuw, A DFT study of the structures, stabilities and redox behaviour of the major surfaces of magnetite $\mathrm{Fe}_{3} \mathrm{O}_{4}$, Phys. Chem. Chem. Phys. 16 (2014) 21082-21097, doi:10.1039/C4CP00529E.
[63] F.D. Murnaghan, The compressibility of media under extreme pressures, Proc. Natl. Acad. Sci. 30 (1944) 244-247, doi:10.1073/pnas.30.9.244.

[64] Y.-R. Jang, B. Deok Yu, Hybrid functional study of the structural and electronic properties of Co and Ni, J. Phys. Soc. Jpn. 81 (2012) 114715, doi:10.1143/JPSJ.81.114715.

[65] Y. Shoaib Mohammed, Y. Yan, H. Wang, K. Li, X. Du, Stability of ferromagnetism in $\mathrm{Fe}$, Co, and ni metals under high pressure with GGA and GGA+U, J. Magn. Magn. Mater. 322 (2010) 653-657, doi:10.1016/j.jmmm.2009.10.033.

[66] J. Wang, S.Q. Wang, Surface energy and work function of fcc and bcc crystals: density functional study, Surf. Sci. 630 (2014) 216-224, doi:10.1016/j.susc.2014.08.017.

[67] J. Lahtinen, J. Vaari, K. Kauraala, Adsorption and structure dependent desorption of CO on Co(0001), Surf. Sci. 418 (1998) 502-510, doi:10.1016/S0039-6028(98)00711-0.

[68] T. Li, B.L. Rickman, W.A. Schroeder, Density functional theory analysis of hexagonal close-packed elemental metal photocathodes, Phys. Rev. Spec. Top. - Accel. Beams 18 (2015) 1-11, doi:10.1103/PhysRevSTAB.18.073401.

[69] J.E. Prieto, C. Rath, S. Müller, R. Miranda, K. Heinz, A structural analysis of the $\mathrm{Co}(0001)$ surface and the early stages of the epitaxial growth of $\mathrm{Cu}$ on it, Surf. Sci. 401 (1998) 248-260, doi:10.1016/S0039-6028(97)01085-6.

[70] B.W. Lee, R. Alsenz, A. Ignatiev, M.A. Van Hove, Surface structures of the two allotropic phases of cobalt, Phys. Rev. B 17 (1978) 1510-1520, doi:10.1103/PhysRevB.17.1510.

[71] W.R. Tyson, W.A. Miller, Surface free energies of solid metals: estimation from liquid surface tension measurements, Surf. Sci. 62 (1977) 267-276, doi:10.1016/0039-6028(77)90442-3.

[72] L. Vitos, A.V. Ruban, H.L. Skriver, J. Kollár, The surface energy of metals, Surf. Sci. 411 (1998) 186-202, doi:10.1016/S0039-6028(98)00363-X.

[73] V.B. Nguyen, M. Benoit, N. Combe, H. Tang, Prediction of co nanoparticle morphologies stabilized by ligands: towards a kinetic model, Phys. Chem. Chem. Phys. 4636 (2017) 4636-4647, doi:10.1039/c6cp08153c.

[74] Q. Ge, M. Neurock, Adsorption and activation of CO over flat and stepped co surfaces: a first principles analysis, J. Phys. Chem. B 110 (2006) 15368-15380, doi:10.1021/jp060477i.

[75] W. Luo, A. Asthagiri, Density functional theory study of methanol steam reforming on Co(0001) and Co(111) surfaces, J. Phys. Chem. C 118 (2014) 15274-15285, doi:10.1021/jp503177h.

[76] X.-Q. Gong, R. Raval, P. Hu, CO dissociation and O removal on Co(0001): a density functional theory study, Surf. Sci. 562 (2004) 247-256, doi:10.1016/j.susc.2004.06.151

[77] S. Schwegmann, A.P. Seitsonen, V. De Renzi, H. Dietrich, H. Bludau, M. Gierer, H. Over, K. Jacobi, M. Scheffler, G. Ertl, Oxygen adsorption on the Ru(1010) surface: anomalous coverage dependence, Phys. Rev. B 57 (1998) 15487-15495, doi:10.1103/PhysRevB.57.15487.

[78] M.E. Bridge, R.M. Lambert, Oxygen chemisorption, surface oxidation, and the oxidation of carbon monoxide on cobalt (0001), Surf. Sci. 82 (1979) 413-424, doi:10.1016/0039-6028(79)90199-7.

[79] B. Lee, A. Ignatiev, J. Taylor, J. Rabalais, Atomic structure sensitivity of XPS: the oxidation of cobalt, Solid State Commun. 33 (1980) 1205-1208, doi:10.1016/0038-1098(80)90791-7.

[80] B. Klingenberg, F. Grellner, D. Borgmann, G. Wedler, Low-energy electron diffraction and X-ray photoelectron spectroscopy on the oxidation of cobalt (1120), Surf. Sci. 383 (1997) 13-24, doi:10.1016/S0039-6028(97)00107-6.

[81] M. Gierer, H. Over, P. Rech, E. Schwarz, K. Christmann, The adsorption geometry of the $(2 \times 1)-2 O$ oxygen phase formed on the Co(1010) surface, Surf. Sci. 370 (1997) L201-L206, doi:10.1016/S0039-6028(96)01202-2.

[82] A.L. Allred, Electronegativity values from thermochemical data, J. Inorg. Nucl. Chem. 17 (1961) 215-221, doi:10.1016/0022-1902(61)80142-5.

[83] F. Grellner, B. Klingenberg, D. Borgmann, G. Wedler, Electron spectroscopic study of the interaction of oxygen with $\mathrm{Co}(110)$ and of coadsorption with water, J. Electron Spectros. Relat. Phenomena. 71 (1995) 107-115, doi:10.1016/0368-2048(94)02261-5.

[84] M.V. Ganduglia-Pirovano, M. Scheffler, Structural and electronic properties of chemisorbed oxygen on Rh(111), Phys. Rev. B 59 (1999) 15533-15543, doi:10.1103/PhysRevB.59.15533.

[85] M. Todorova, K. Reuter, M. Scheffler, Oxygen overlayers on Pd(111) studied by density functional theory, J. Phys. Chem. B 108 (2004) 14477-14483, doi:10.1021/jp040088t.

[86] B. Sun, P. Zhang, Z. Wang, S. Duan, X.G. Zhao, X. Ma, Q.K. Xue, Atomic oxygen adsorption and incipient oxidation of the $\mathrm{Pb}(111)$ surface: a density-functional theory study, Phys. Rev. B - Condens. Matter Mater. Phys. 78 (2008) 1-12, doi:10.1103/PhysRevB.78.035421.

[87] G. Henkelman, A. Arnaldsson, H. Jónsson, A fast and robust algorithm for bader decomposition of charge density, Comput. Mater. Sci. 36 (2006) 354-360, doi:10.1016/j.commatsci.2005.04.010.

[88] S. Maintz, V.L. Deringer, A.L. Tchougréeff, R. Dronskowski, LOBSTER: a tool to extract chemical bonding from plane-wave based DFT, J. Comput. Chem. 37 (2016) 1030-1035, doi:10.1002/jcc.24300.

[89] M.E. Bridge, R.M. Lambert, Oxygen chemisorption, surface oxidation, and the oxidation of carbon monoxide on cobalt (0001), Surf. Sci. 82 (1979) 413-424, doi:10.1016/0039-6028(79)90199-7.

[90] R.B. Moyes, M.W. Roberts, Interaction of cobalt with oxygen, water vapor, and carbon monoxide. X-Ray and ultraviolet photoemission studies, J. Catal. 49 (1977) 216224, doi:10.1016/0021-9517(77)90257-3.

[91] S. Mrowec, K. Przybylski, Oxidation of cobalt at high temperature, Oxid. Met. 11 (1977) 365-381, doi:10.1007/BF00608018. 